\title{
Disjoint Paths in Graphs III, Characterization
}

\author{
Xingxing $\mathrm{Yu}^{*}$ \\ School of Mathematics \\ Georgia Institute of Technology \\ Atlanta, GA 30332
}

\begin{abstract}
Let $G$ be a graph, $\{a, b, c\} \subseteq V(G)$, and $\left\{a^{\prime}, b^{\prime}, c^{\prime}\right\} \subseteq V(G)$ such that $\{a, b, c\} \neq$ $\left\{a^{\prime}, b^{\prime}, c^{\prime}\right\}$. We say that $\left(G,\{a, c\},\left\{a^{\prime}, c^{\prime}\right\},\left(b, b^{\prime}\right)\right)$ is an obstruction if, for any three vertex disjoint paths from $\{a, b, c\}$ to $\left\{a^{\prime}, b^{\prime}, c^{\prime}\right\}$ in $G$, one path is from $b$ to $b^{\prime}$. In this paper we characterize obstructions.
\end{abstract}

${ }^{*}$ Partially supported by NSF grant DMS-9970527 and NSA grant MDA904-03-1-0052 


\section{Introduction}

We use the terminology in [5]. Let $G$ be a graph, $\{a, b, c\} \subseteq V(G)$, and $\left\{a^{\prime}, b^{\prime}, c^{\prime}\right\} \subseteq$ $V(G)$. Then $\left(G,\{a, c\},\left\{a^{\prime}, c^{\prime}\right\},\left(b, b^{\prime}\right)\right)$ is an obstruction if for any three vertex disjoint paths from $\{a, b, c\}$ to $\left\{a^{\prime}, b^{\prime}, c^{\prime}\right\}$ in $G$, one path is from $b$ to $b^{\prime}$. In [6], a special class of obstructions are characterized. In this paper, we characterize all obstructions. In order to state our main result, we need the following definition from [5].

A 3-planar graph $(G, \mathcal{A})$ consists of a graph $G$ and a set $\mathcal{A}=\left\{A_{1}, \ldots, A_{k}\right\}$ of pairwise disjoint subsets of $V(G)$ (possibly $\mathcal{A}=\emptyset$ ) such that

(a) for $1 \leq i \neq j \leq k, N\left(A_{i}\right) \cap A_{j}=\emptyset$,

(b) for $1 \leq i \leq k,\left|N\left(A_{i}\right)\right| \leq 3$, and

(c) if $p(G, \mathcal{A})$ denotes the graph obtained from $G$ by (for each $i$ ) deleting $A_{i}$ and adding edges joining every pair of distinct vertices in $N\left(A_{i}\right)$, then $p(G, \mathcal{A})$ can be drawn in a closed disc $D$ with no pair of edges crossing such that, for each $A_{i}$ with $\left|N\left(A_{i}\right)\right|=3, N\left(A_{i}\right)$ induces a facial triangle in $p(G, \mathcal{A})$.

If, in addition, $b_{0}, b_{1}, \ldots, b_{n}$ are vertices of $G$ such that $b_{i} \notin A_{j}$ for any $A_{j} \in \mathcal{A}$ and $b_{0}, b_{1}, \ldots, b_{n}$ occur on the boundary of $D$ in that cyclic order, then we say that $\left(G, \mathcal{A}, b_{0}, b_{1}, \ldots, b_{n}\right)$ is 3-planar. If there is no need to specify $\mathcal{A}$, we will simply say that $\left(G, b_{0}, b_{1}, \ldots, b_{n}\right)$ is 3-planar.

The building blocks of obstructions are described in the following definition.

(1.1) Definition. Let $G$ be a graph, $\{a, b, c\} \subseteq V(G)$, and $\left\{a^{\prime}, b^{\prime}, c^{\prime}\right\} \subseteq V(G)$. Suppose $\{a, b, c\} \neq\left\{a^{\prime}, b^{\prime}, c^{\prime}\right\}$, and assume that $G$ has no 3 -separation $\left(G_{1}, G_{2}\right)$ such that $\{a, b, c\} \subseteq G_{1}$ and $\left\{a^{\prime}, b^{\prime}, c^{\prime}\right\} \subseteq G_{2}$. Then we call $\left(G,(a, b, c),\left(a^{\prime}, b^{\prime}, c^{\prime}\right)\right)$ a rung if one of the following conditions is satisfied:

(1) $b=b^{\prime}$ or $\{a, c\}=\left\{a^{\prime}, c^{\prime}\right\}$;

(2) $a=a^{\prime}$ and $\left(G-a, c, c^{\prime}, b^{\prime}, b\right)$ is 3-planar;

$\left(2^{\prime}\right) c=c^{\prime}$ and $\left(G-c, a, a^{\prime}, b^{\prime}, b\right)$ is 3-planar;

(3) $\{a, b, c\} \cap\left\{a^{\prime}, b^{\prime}, c^{\prime}\right\}=\emptyset$ and $\left(G, a^{\prime}, b^{\prime}, c^{\prime}, c, b, a\right)$ is 3-planar;

(4) $\{a, b, c\} \cap\left\{a^{\prime}, b^{\prime}, c^{\prime}\right\}=\emptyset, G$ has a 1-separation $\left(G_{1}, G_{2}\right)$ such that $\left\{a, a^{\prime}, b, b^{\prime}\right\} \subseteq G_{1}$, $\left\{c, c^{\prime}\right\} \subseteq G_{2}$, and $\left(G_{1}, a, a^{\prime}, b^{\prime}, b\right)$ is 3-planar;

$\left(4^{\prime}\right)\{a, b, c\} \cap\left\{a^{\prime}, b^{\prime}, c^{\prime}\right\}=\emptyset, G$ has a 1-separation $\left(G_{1}, G_{2}\right)$ such that $\left\{c, c^{\prime}, b, b^{\prime}\right\} \subseteq G_{1}$, $\left\{a, a^{\prime}\right\} \subseteq G_{2}$, and $\left(G_{1}, c, c^{\prime}, b^{\prime}, b\right)$ is 3-planar;

(5) $\{a, b, c\} \cap\left\{a^{\prime}, b^{\prime}, c^{\prime}\right\}=\emptyset,\left(G, a, a^{\prime}, b^{\prime}, b\right)$ is 3-planar, and $G$ has a separation $\left(G_{1}, G_{2}\right)$ such that $V\left(G_{1} \cap G_{2}\right)=\{z, b\}$ (or $\left.V\left(G_{1} \cap G_{2}\right)=\left\{z, b^{\prime}\right\}\right),\left\{a, a^{\prime}, b, b^{\prime}\right\} \subseteq G_{1}$, $\left\{c, c^{\prime}\right\} \subseteq G_{2}$, and $\left(G_{2}, c, c^{\prime}, z, b\right)$ (or $\left.\left(G_{2}, c, c^{\prime}, b^{\prime}, z\right)\right)$ is 3-planar; 
$\left(5^{\prime}\right)\{a, b, c\} \cap\left\{a^{\prime}, b^{\prime}, c^{\prime}\right\}=\emptyset,\left(G, c, c^{\prime}, b^{\prime}, b\right)$ is 3-planar, and $G$ has a separation $\left(G_{1}, G_{2}\right)$ such that $V\left(G_{1} \cap G_{2}\right)=\{z, b\}$ (or $\left.V\left(G_{1} \cap G_{2}\right)=\left\{z, b^{\prime}\right\}\right),\left\{c, c^{\prime}, b, b^{\prime}\right\} \subseteq G_{1},\left\{a, a^{\prime}\right\} \subseteq$ $G_{2}$, and $\left(G_{2}, a, a^{\prime}, z, b\right)$ (or $\left.\left(G_{2}, a, a^{\prime}, b^{\prime}, z\right)\right)$ is 3-planar;

(6) $\{a, b, c\} \cap\left\{a^{\prime}, b^{\prime}, c^{\prime}\right\}=\emptyset$, and there are pairwise edge disjoint subgraphs $G_{a}, G_{c}, M$ of $G$ such that $G=G_{a} \cup G_{c} \cup M, V\left(G_{a} \cap M\right)=\{u, w\}, V\left(G_{c} \cap M\right)=\{p, q\}$, $V\left(G_{a} \cap G_{c}\right)=\emptyset,\left\{a, a^{\prime}, b^{\prime}\right\} \subseteq G_{a},\left\{c, c^{\prime}, b\right\} \subseteq G_{c},\left(G_{a}, a, a^{\prime}, b^{\prime}, w, u\right)$ is 3-planar, and $\left(G_{c}, c^{\prime}, c, b, p, q\right)$ is 3-planar;

$\left(6^{\prime}\right)\{a, b, c\} \cap\left\{a^{\prime}, b^{\prime}, c^{\prime}\right\}=\emptyset$, and there are pairwise edge disjoint subgraphs $G_{a}, G_{c}, M$ of $G$ such that $G=G_{a} \cup G_{c} \cup M, V\left(G_{a} \cap M\right)=\{u, w\}, V\left(G_{c} \cap M\right)=\{p, q\}$, $V\left(G_{a} \cap G_{c}\right)=\emptyset,\left\{a, a^{\prime}, b\right\} \subseteq G_{a},\left\{c, c^{\prime}, b^{\prime}\right\} \subseteq G_{c},\left(G_{a}, b, a, a^{\prime}, w, u\right)$ is 3-planar, and $\left(G_{c}, b^{\prime}, c^{\prime}, c, p, q\right)$ is 3-planar;

(7) $\{a, b, c\} \cap\left\{a^{\prime}, b^{\prime}, c^{\prime}\right\}=\emptyset$, and there are pairwise edge disjoint subgraphs $G_{a}, G_{c}, M$ of $G$ such that $G=G_{a} \cup G_{c} \cup M, V\left(G_{a} \cap M\right)=\left\{b, b^{\prime}, w\right\}, V\left(G_{c} \cap M\right)=\left\{b, b^{\prime}, p\right\}$, $V\left(G_{a} \cap G_{c}\right)=\left\{b, b^{\prime}\right\},\left\{a, a^{\prime}\right\} \subseteq G_{a},\left\{c, c^{\prime}\right\} \subseteq G_{c},\left(G_{a}, a, a^{\prime}, b^{\prime}, w, b\right)$ is 3-planar, and $\left(G_{c}, c^{\prime}, c, b, p, b^{\prime}\right)$ is 3 -planar.

(1.2) Definition. Let $L$ be a graph and let $R_{1}, \ldots, R_{m}$ be edge disjoint subgraphs of $L$ such that

(i) $\left(R_{i},\left(x_{i-1}, v_{i-1}, y_{i-1}\right),\left(x_{i}, v_{i}, y_{i}\right)\right)$ is a rung for each $1 \leq i \leq m$,

(ii) $V\left(R_{i} \cap R_{j}\right)=\left\{x_{i}, v_{i}, y_{i}\right\} \cap\left\{x_{j-1}, v_{j-1}, y_{j-1}\right\}$ for $1 \leq i<j \leq m$,

(iii) for any $1 \leq i<j \leq m$, if $x_{i}=x_{j}$ then $x_{k}=x_{i}$ for all $i \leq k \leq j$, if $v_{i}=v_{j}$ then $v_{k}=v_{i}$ for all $i \leq k \leq j$, and if $y_{i}=y_{j}$ then $y_{k}=y_{i}$ for all $i \leq k \leq j$.

(iv) $L=\left(\bigcup_{i=1}^{m} R_{i}\right)+S$, where $S$ consists of edges of $L$ with both ends in some $\left\{x_{i}, v_{i}, y_{i}\right\}$, $1 \leq i \leq m$.

Then we call $\left(L,\left(x_{0}, v_{0}, y_{0}\right),\left(x_{m}, v_{m}, y_{m}\right)\right)$ a ladder with rungs $\left(R_{i},\left(x_{i-1}, v_{i-1}, y_{i-1}\right)\right.$, $\left.\left(x_{i}, v_{i}, y_{i}\right)\right), i=1, \ldots, m$, or simply, a ladder along $v_{0} \ldots v_{m}$.

It is easy to see that a rung is at most 5-connected. It was shown in [5] (Proposition 4.4) that if $\left(L,\left(x_{0}, v_{0}, y_{0}\right),\left(x_{m}, v_{m}, y_{m}\right)\right)$ is a ladder, then $\left(L,\left\{x_{0}, y_{0}\right\},\left\{x_{m}, y_{m}\right\},\left(v_{0}, v_{m}\right)\right)$ is an obstruction. The main result of this paper states that every obstruction can be constructed from ladders and 3-planar graphs in a special way. For a sequence $S$, the reduced sequence of $S$ is the sequence obtained from $S$ by removing all but one consecutive identical elements. For example, the reduced sequence of aaabcca is abca.

(1.3) Theorem. Let $G$ be a graph, $\{a, b, c\} \subseteq V(G)$, and $\left\{a^{\prime}, b^{\prime}, c^{\prime}\right\} \subseteq V(G)$ such that $\{a, b, c\} \neq\left\{a^{\prime}, b,^{\prime} c^{\prime}\right\}$. Assume that, for any $T \subseteq V(G)$ with $|T| \leq 3$, every component of $G-T$ contains some element of $\{a, b, c\} \cup\left\{a^{\prime}, b^{\prime}, c^{\prime}\right\}$. Then $\left(G,\{a, c\},\left\{a^{\prime}, c^{\prime}\right\},\left(b, b^{\prime}\right)\right)$ is an obstruction iff one of the following statements holds. 
(1) $G$ has a separation $\left(G_{1}, G_{2}\right)$ of order at most 2 such that $\{a, b, c\} \subseteq V\left(G_{1}\right)$ and $\left\{a^{\prime}, b^{\prime}, c^{\prime}\right\} \subseteq V\left(G_{2}\right)$.

(2) $\left(G,(a, b, c),\left(a^{\prime}, b^{\prime}, c^{\prime}\right)\right)$ is a ladder.

(3) $G$ has a separation $(J, L)$ such that $V(J \cap L)=\left\{w_{0}, \ldots, w_{n}\right\},\left(J, w_{0}, \ldots, w_{n}\right)$ is 3-planar, $\left(L,(a, b, c),\left(a^{\prime}, b^{\prime}, c^{\prime}\right)\right)$ is a ladder along a sequence $v_{0} \ldots v_{m}$, where $v_{0}=b$, $v_{m}=b^{\prime}$, and $w_{0} \ldots w_{n}$ is the reduced sequence of $v_{0} \ldots v_{m}$.

Note the condition of (1.3) that for any $T \subseteq V(G)$ with $|T| \leq 3$, every component of $G-T$ contains some element of $\{a, b, c\} \cup\left\{a^{\prime}, b^{\prime}, c^{\prime}\right\}$. It is a natural condition, for the following reason. Suppose that $T \subseteq V(G),|T| \leq 3$, and $G-T$ contains a component $H$ with $V(H) \cap\left(\{a, b, c\} \cup\left\{a^{\prime}, b^{\prime}, c^{\prime}\right\}\right)=\emptyset$. Let $G^{\prime}$ be obtained from $G$ by removing $H$ and adding new edges between every pair of distinct vertices in $N(H)$. Then it is easy to see that $\left(G,\{a, c\},\left\{a^{\prime}, c^{\prime}\right\},\left(b, b^{\prime}\right)\right)$ is an obstruction iff $\left(G^{\prime},\{a, c\},\left\{a^{\prime}, c^{\prime}\right\},\left(b, b^{\prime}\right)\right)$ is an obstruction.

Also note that if (1) of (1.3) holds, then there do not exist three disjoint paths from $\{a, b, c\}$ to $\left\{a^{\prime}, b^{\prime}, c^{\prime}\right\}$, and hence, $\left(G,\{a, c\},\left\{a^{\prime}, c^{\prime}\right\},\left(b, b^{\prime}\right)\right)$ is an obstruction in a trivial sense.

As a consequence of (1.3), we will prove the following result.

(1.4) Corollary. Let $\left(G,\{a, c\},\left\{a^{\prime}, c^{\prime}\right\},\left(b, b^{\prime}\right)\right)$ be an obstruction. If $\{a, c\} \neq\left\{a^{\prime}, c^{\prime}\right\}$ and $b \neq b^{\prime}$, then $G$ is at most 7-connected.

The rest of the paper is organized as follows. In Section 2, we prove a technical lemma. In Section 3, we prove (1.3). In Section 4, we will prove (1.4) and construct 7-connected obstructions.

\section{Good ladders}

The main goal of this section is to prove a technical lemma, based on good ladders. To this end, we need the following result proved in [2] (also independently in [1] and [3]). Also, see (2.4) of [5].

(2.1) Theorem. Let $G$ be a graph and $\left\{s_{1}, s_{2}, t_{1}, t_{2}\right\} \subseteq V(G)$. Then $G$ contains no $\left(\left\{s_{1}, t_{1}\right\},\left\{s_{2}, t_{2}\right\}\right)$-linkage iff $\left(G, s_{1}, s_{2}, t_{1}, t_{2}\right)$ is 3-planar.

We also need the following result, which follows easily from Proposition 3.2 of [5].

(2.2) Lemma. Let $(G, \mathcal{A})$ be 3-planar and let $b, b^{\prime} \in V(G)$ be distinct. Let $u, v \in V(G)$ such that $u, v \in p(G, \mathcal{A})$. If $p(G, \mathcal{A})$ contains a $\left(\{u, b\},\left\{v, b^{\prime}\right\}\right)$-linkage, then $G$ contains a $\left(\{u, b\},\left\{v, b^{\prime}\right\}\right)$-linkage $L$. 
To prove the main result of this section, we need the main result of [6]. For convenience, we state the following definition.

(2.3) Definition. Let $\left(G,(a, b, c),\left(a^{\prime}, b^{\prime}, c^{\prime}\right)\right)$ be a rung. Suppose that $G-\left\{b, b^{\prime}\right\}$ has disjoint paths $A, C$ from $a, c$ to $a^{\prime}, c^{\prime}$, respectively, and assume that the following conditions are satisfied:

(1) If $G$ is connected and $b \neq b^{\prime}$, then $G-\left(\left(A-a^{\prime}\right) \cup(C-c)\right)$ contains a $\left(\left\{b, a^{\prime}\right\},\left\{c, b^{\prime}\right\}\right)$ linkage, and $G-\left((A-a) \cup\left(C-c^{\prime}\right)\right)$ contains an $\left(\left\{a, b^{\prime}\right\},\left\{b, c^{\prime}\right\}\right)$-linkage;

$\left(1^{\prime}\right)$ if $G$ is connected and $b=b^{\prime}$, then $G-((A-a) \cup C)$ has a path from $a$ to $b^{\prime}$, $G-\left(\left(A-a^{\prime}\right) \cup C\right)$ has a path from $a^{\prime}$ to $b, G-(A \cup(C-c))$ has a path from $c$ to $b^{\prime}$, and $G-\left(A \cup\left(C-c^{\prime}\right)\right)$ has a path from $c^{\prime}$ to $b$;

(2) if $G$ is not connected and $\{a, b\} \cup\left\{a^{\prime}, b^{\prime}\right\}$ is contained in a component of $G$, then $G-((A-a) \cup C)$ contains a path from $a$ to $b^{\prime}$ (and not using $b$ if $\left.b \neq b^{\prime}\right)$ and $G-\left(\left(A-a^{\prime}\right) \cup C\right)$ contains a path from $b$ to $a^{\prime}$ (and not using $b^{\prime}$ if $b \neq b^{\prime}$ ); and

$\left(2^{\prime}\right)$ if $G$ is not connected and $\{b, c\} \cup\left\{b^{\prime}, c^{\prime}\right\}$ is contained in a component of $G$, then $G-(A \cup(C-c))$ contains a path from $c$ to $b^{\prime}$ (and not using $b$ if $b \neq b^{\prime}$ ), and $G-\left(A \cup\left(C-c^{\prime}\right)\right)$ contains a path from $b$ to $c^{\prime}$ (and not using $b^{\prime}$ if $b \neq b^{\prime}$ ).

Then we call $\left(G,(a, b, c),\left(a^{\prime}, b^{\prime}, c^{\prime}\right), A, C\right)$ a good rung. Let $\left(L,\left(x_{0}, v_{0}, y_{0}\right),\left(x_{m}, v_{m}, y_{m}\right)\right)$ be a ladder with good rungs $\left(R_{i},\left(x_{i-1}, v_{i-1}, y_{i-1}\right),\left(x_{i}, v_{i}, y_{i}\right), X_{i}, Y_{i}\right), i=1, \ldots, m$. Then we say that $\left(L,\left(x_{0}, v_{0}, y_{0}\right),\left(x_{m}, v_{m}, y_{m}\right), X, Y\right)$ is a good ladder along $v_{0} \ldots v_{m}$, where $X=\bigcup_{i=1}^{m} X_{i}$ and $Y=\bigcup_{i=1}^{m} Y_{i}$.

The following is the main result of $[6]$.

(2.4) Lemma. Let $\left(G,\{a, c\},\left\{a^{\prime}, c^{\prime}\right\},\left(b, b^{\prime}\right)\right)$ be an obstruction satisfying the following conditions:

(i) $G-\left\{b, b^{\prime}\right\}$ contains disjoint paths $A, C$ from $a, c$ to $a^{\prime}, c^{\prime}$, respectively,

(ii) $A \cup C$ is an induced subgraph of $G$, and

(iii) $G-(A \cup C)$ is connected.

Then one of the following statements holds:

(1) There is a separation $\left(G_{1}, G_{2}\right)$ in $G$ of order at most 2 such that $\{a, b, c\} \subseteq V\left(G_{1}\right)$ and $\left\{a^{\prime}, b^{\prime}, c^{\prime}\right\} \subseteq V\left(G_{2}\right)$.

(2) There is a subset $T \subseteq V(G)$ such that $|T| \leq 3$ and some component of $G-T$ contains no element of $\{a, b, c\} \cup\left\{a^{\prime}, b^{\prime}, c^{\prime}\right\}$.

(3) $\left(G,(a, b, c),\left(a^{\prime}, b^{\prime}, c^{\prime}\right), A, C\right)$ is a good ladder.

(4) $G$ has a separation $(J, L)$ such that $V(J \cap L)=\left\{w_{0}, \ldots, w_{n}\right\},\left(J, w_{0}, \ldots, w_{n}\right)$ is 3-planar, $\left(L,(a, b, c),\left(a^{\prime}, b^{\prime}, c^{\prime}\right), A, C\right)$ is a good ladder along a sequence $v_{0} \ldots v_{m}$, where $v_{0}=b, v_{m}=b^{\prime}$, and $w_{0} \ldots w_{n}$ is the reduced sequence of $v_{0} \ldots v_{m}$. 
We can now state and prove the technical lemma mentioned earlier. The conditions of the lemma arise in the proof of (1.3).

(2.5) Lemma. Let $\left(L,(a, b, c),\left(a^{\prime}, b^{\prime}, c^{\prime}\right), A, C\right)$ be a good ladder with good rungs $\left(R_{i},\left(x_{i-1}, v_{i-1}, y_{i-1}\right),\left(x_{i}, v_{i}, y_{i}\right), A_{i}, C_{i}\right)$, where $A_{i}=A \cap R_{i}, C_{i}=C \cap C_{i}, a=x_{0}$, $a^{\prime}=x_{m}, b=v_{0}, b^{\prime}=v_{m}, c=y_{0}$, and $c^{\prime}=y_{m}$. Let $L^{\prime}$ be a graph and let $L^{*}$ be obtained from the disjoint union of $L$ and $L^{\prime}$ by adding edges between $V(A \cup C)$ and $V\left(L^{\prime}\right)$ and between $V(A)$ and $V(C)$. Assume the following conditions hold. (We use $N$ instead of $N_{L^{*}}$ to denote neighborhood in $L^{*}$.)

(a) For any $i, j \in\{1, \ldots, m\}$ with $i \leq j$, let $L_{i, j}$ denote the graph obtained from $L^{*}$ by deleting all $R_{k}-\left(A_{k} \cup C_{k} \cup\left\{v_{i-1}, v_{j}\right\}\right)$ with $k<i$ or $k>j$ and by deleting $v_{0}, \ldots, v_{m}$ except $v_{i-1}$ and $v_{j}$. Then $\left(L_{i, j},\{a, c\},\left\{a^{\prime}, c^{\prime}\right\},\left(v_{i-1}, v_{j}\right)\right)$ is an obstruction.

(b) If $q \in V(A)$ (respectively, $q \in V(C)$ ) such that $L$ contains a path from $q$ to $\left\{v_{0}, \ldots, v_{m}\right\}$ internally disjoint from $V(A \cup C) \cup\left\{v_{0}, \ldots, v_{m}\right\}$, then, for any component $D$ of $L^{\prime}, N(D) \cap V(A) \subseteq a A q$ or $N(D) \cap V(A) \subseteq q A a^{\prime}$ (respectively, $N(D) \cap V(C) \subseteq c C q$ or $\left.N(D) \cap V(C) \subseteq q C c^{\prime}\right)$.

(c) If $L^{*}$ contains paths from distinct $s, s^{\prime} \in V(A)$ (in that order from a to $a^{\prime}$ ) to distinct $t, t^{\prime} \in V(C)$ (in that order from $c^{\prime}$ to $c$ ), respectively, which are internally disjoint from $L$, then $L$ contains no path from $V\left(s A s^{\prime}-\left\{s, s^{\prime}\right\}\right) \cup V\left(t C t^{\prime}-\left\{t, t^{\prime}\right\}\right)$ to $\left\{v_{0}, \ldots, v_{m}\right\}$ internally disjoint from $V(A \cup C) \cup\left\{v_{0}, \ldots, v_{m}\right\}$.

(d) Suppose that $L^{*}$ contains paths from $s, s^{\prime} \in V(A)$ (in that order from a to $a^{\prime}$ ) to $t, t^{\prime} \in V(C)$ (in that order from $c$ to $c^{\prime}$ ), respectively, which are internally disjoint from $L$. If $L$ contains a path from $V\left(t C t^{\prime}-\left\{t, t^{\prime}\right\}\right)$ (respectively, $V\left(s A s^{\prime}-\left\{s, s^{\prime}\right\}\right)$ ) to $\left\{v_{0}, \ldots, v_{m}\right\}$ internally disjoint from $V(A \cup C) \cup\left\{v_{0}, \ldots, v_{m}\right\}$, then there is a vertex $q \in s A s^{\prime}$ (respectively, $q \in t C t^{\prime}$ ) such that, for every component $D$ of $L^{\prime}$, $N(D) \cap V(A) \subseteq a A q$ or $N(D) \cap V(A) \subseteq q A a^{\prime}$ (respectively, $N(D) \cap V(C) \subseteq c C q$ or $\left.N(D) \cap V(C) \subseteq q C c^{\prime}\right)$.

(e) If $R_{i}$ is not connected, but $\left\{x_{i-1}, v_{i-1}\right\} \cup\left\{x_{i}, v_{i}\right\}$ (respectively, $\left\{y_{i-1}, v_{i-1}\right\} \cup\left\{y_{i}, v_{i}\right\}$ ) is contained in a component of $R_{i}$, then $v_{i-1}=v_{i}$.

(f) For any $T \subseteq V\left(L^{*}\right)$ with $|T| \leq 3$, every component of $L^{*}-T$ contains a vertex in $V(A \cup C) \cup\left\{v_{0}, \ldots, v_{m}\right\}$.

Then $\left(L^{*},(a, b, c),\left(a^{\prime}, b^{\prime}, c^{\prime}\right)\right)$ is a ladder along a sequence $z_{0} \ldots z_{p}$, and the reduced sequence of $z_{0} \ldots z_{p}$ is the reduced sequence of $v_{0} \ldots v_{m}$. 
Proof. Suppose (2.5) fails. Choose $L^{*}, L, A, C$ so that $\left|V\left(L^{*}\right)\right|+\left|E\left(L^{*}\right)\right|$ is minimum. Then it is easy to see that $\{a, b, c\}$ and $\left\{a^{\prime}, b^{\prime}, c^{\prime}\right\}$ are independent sets in $L^{*}$. We proceed by proving Claims $1-6$.

Claim 1. $L^{*}$ does not contain any separation $\left(G_{1}, G_{2}\right)$ such that $V\left(G_{1} \cap G_{2}\right)=$ $\left\{a^{\prime \prime}, v_{k}, c^{\prime \prime}\right\},\{a, b, c\} \subseteq G_{1}$, and $\left\{a^{\prime}, b^{\prime}, c^{\prime}\right\} \subseteq G_{2}$, where $k \in\{0, \ldots, m\}, a^{\prime \prime} \in V(A)$, and $c^{\prime \prime} \in V(C)$.

Suppose that such a separation $\left(G_{1}, G_{2}\right)$ does exist. Note that $\left\{a^{\prime \prime}, v_{k}, c^{\prime \prime}\right\} \neq\{a, b, c\}$ and $\left\{a^{\prime \prime}, v_{k}, c^{\prime \prime}\right\} \neq\left\{a^{\prime}, b^{\prime}, c^{\prime}\right\}$; for otherwise, since $\{a, b, c\}$ and $\left\{a^{\prime}, b^{\prime}, c^{\prime}\right\}$ are independent in $L^{*}, L^{*}-\left\{a^{\prime \prime}, v_{k}, c^{\prime \prime}\right\}$ has a component not containing any element of $V(A) \cup V(C) \cup$ $\left\{v_{0}, \ldots, v_{m}\right\}$, contradicting (f).

For $i \in\{1,2\}$, let $L_{i}=L \cap G_{i}, L_{i}^{\prime}=L^{\prime} \cap G_{i}, X_{i}=A \cap G_{i}$ and $Y_{i}=C \cap G_{i}$.

First, we claim that $\left(L_{1},(a, b, c),\left(a^{\prime \prime}, v_{k}, c^{\prime \prime}\right), X_{1}, Y_{1}\right)$ is a good ladder along a sequence whose reduced sequence is the reduced sequence of $v_{0} \ldots v_{k}$. This is clear if, for every $i \in\{1, \ldots, m\}$, either $R_{i} \subseteq L_{1}$ or $R_{i} \subseteq L_{2}$. So assume that $p$ is minimum such that $R_{p} \nsubseteq L_{1}$ and $R_{p} \nsubseteq L_{2}$, and $q$ is maximum such that $R_{q} \nsubseteq L_{1}$ and $R_{q} \nsubseteq L_{2}$. Then, for each $p \leq i \leq q,\left\{a_{i-1}, v_{i-1}, c_{i-1}\right\} \nsubseteq L_{2}$ and $\left\{a_{i}, v_{i}, c_{i}\right\} \nsubseteq L_{1}$, and hence, $R_{i} \nsubseteq L_{1}$ and $R_{i} \nsubseteq L_{2}$. Let $R_{i}^{1}=R_{i}$ for $1 \leq i<p$; then $\left(R_{i}^{1},\left(x_{i-1}, v_{i-1}, y_{i-1}\right),\left(x_{i}, v_{i}, y_{i}\right), A_{i}, C_{i}\right)$ is a good rung. Now assume $i \in\{p, \ldots, q\}$. Because $R_{i}$ has no 3-separation $\left(R_{i}^{\prime}, R_{i}^{\prime \prime}\right)$ such that $\left\{x_{i-1}, v_{i-1}, y_{i-1}\right\} \subseteq R_{i}^{\prime}$ and $\left\{x_{i}, v_{i}, y_{i}\right\} \subseteq R_{i}^{\prime \prime}$, either $x_{i-1} A x_{i} \subseteq a A a^{\prime \prime}$ and $y_{i-1} C y_{i} \subseteq c^{\prime \prime} C c^{\prime}$ or $x_{i-1} A x_{i} \subseteq a^{\prime \prime} A a^{\prime}$ and $y_{i-1} C y_{i} \subseteq c C c^{\prime \prime}$. This implies that $a^{\prime \prime} \in$ $\left\{x_{p-1}, \ldots, x_{q}\right\}$ and $c^{\prime \prime} \in\left\{y_{p-1}, \ldots, y_{q}\right\}$. Let $R_{i}^{1}=\left(R_{i} \cap L_{1}\right) \cup\left\{c^{\prime \prime}\right\}$ if $x_{i-1} A x_{i} \subseteq a A a^{\prime \prime}$, and let $R_{i}^{1}=\left(R_{i} \cap L_{1}\right) \cup\left\{a^{\prime \prime}\right\}$ if $y_{i-1} C y_{i} \subseteq c C c^{\prime \prime}$. Note that $R_{i}^{1}$ is not connected. Since $\left(R_{i},\left(x_{i-1}, v_{i-1}, y_{i-1}\right),\left(x_{i}, v_{i}, y_{i}\right), A_{i}, C_{i}\right)$ is a good rung, it is easy to see that (by using (2.3)) if $x_{i-1} A x_{i} \subseteq a A a^{\prime \prime}$ then $\left(R_{i}^{1},\left(x_{i-1}, v_{i-1}, c^{\prime \prime}\right),\left(x_{i}, v_{k}, c^{\prime \prime}\right), A_{i},\left\{c^{\prime \prime}\right\}\right)$ is a good rung, and if $y_{i-1} C y_{i} \subseteq c C c^{\prime \prime}$ then $\left(R_{i}^{1},\left(a^{\prime \prime}, v_{i-1}, y_{i-1}\right),\left(a^{\prime \prime}, v_{k}, y_{i}\right),\left\{a^{\prime \prime}\right\}, C_{i}\right)$ is a good rung. Hence, $\left(L_{1},(a, b, c),\left(a^{\prime \prime}, v_{k}, c^{\prime \prime}\right), X_{1}, Y_{1}\right)$ is a good ladder along a sequence whose reduced sequence is the reduced sequence of $v_{0} \ldots v_{k}$.

Similarly, we can show that $\left(L_{2},\left(a^{\prime \prime}, v_{k}, c^{\prime \prime}\right),\left(a^{\prime}, b^{\prime}, c^{\prime}\right), X_{2}, Y_{2}\right)$ is a good ladder along a sequence whose reduced sequence is the reduced sequence of $v_{k} \ldots v_{m}$.

It is easy to see that, for $i \in\{1,2\}, G_{i}, L_{i}, L_{i}^{\prime}, X_{i}, Y_{i}$ (as $L^{*}, L, L^{\prime}, A, C$, respectively) also satisfy (a)-(f) of (2.5) (with $a^{\prime \prime}, v_{k}, c^{\prime \prime}$ as $a^{\prime}, b^{\prime}, c^{\prime}$, respectively, when $i=1$, and with $a^{\prime \prime}, b^{\prime \prime}, c^{\prime \prime}$ as $a, b, c$, respectively, when $\left.i=2\right)$.

Since $\left|V\left(G_{1}\right)\right|+\left|E\left(G_{1}\right)\right|<\left|V\left(L^{*}\right)\right|+\left|E\left(G^{*}\right)\right|,\left(G_{1},(a, b, c),\left(a^{\prime \prime}, v_{k}, c^{\prime \prime}\right)\right)$ is a ladder along $z_{0} \ldots z_{q}$, where $z_{0}=v_{0}$ and $z_{q}=v_{k}$, and the reduced sequence of $z_{0} \ldots z_{q}$ is the reduced sequence of $v_{0} \ldots v_{k}$. Similarly, $\left(G_{2},\left(a^{\prime \prime}, v_{k}, c^{\prime \prime}\right),\left(a^{\prime}, b^{\prime}, c^{\prime}\right)\right)$ is a ladder along $z_{q} \ldots z_{p}$, where $z_{q}=v_{k}$ and $z_{p}=v_{m}$, and the reduced sequence of $z_{q} \ldots z_{p}$ is the reduced sequence of $v_{k} \ldots v_{m}$. Hence, $\left(L^{*},(a, b, c),\left(a^{\prime}, b^{\prime}, c^{\prime}\right)\right)$ is a ladder along $z_{0} \ldots z_{p}$, where $z_{0}=v_{0}=b$ and $z_{p}=v_{m}=b^{\prime}$, and the reduced sequence of $z_{0} \ldots z_{p}$ is the reduced sequence of $v_{0} \ldots v_{m}$. This is a contradiction. 
Claim 2. $b \neq b^{\prime}$ and $\{a, c\} \neq\left\{a^{\prime}, c^{\prime}\right\}$.

Suppose $b=b^{\prime}$ or $\{a, c\}=\left\{a^{\prime}, c^{\prime}\right\}$. Then by Claim $1,\left(L^{*},(a, b, c),\left(a^{\prime}, b^{\prime}, c^{\prime}\right)\right)$ is a rung (as in (1) of (1.1)), and so, a ladder, contradicting the choice of $L^{*}$.

Claim 3. (1) If $\left\{x_{i-1}, v_{i-1}\right\} \cup\left\{x_{i}, v_{i}\right\}$ is contained in a component of $R_{i}$, then for each $x \in\left\{x_{i-1}, x_{i}\right\}$ and for every component $D$ of $L^{\prime}, N(D) \cap V(A) \subseteq a A x$ or $N(D) \cap$ $V(A) \subseteq x A a^{\prime}$. (2) If $\left\{y_{i-1}, v_{i-1}\right\} \cup\left\{y_{i}, v_{i}\right\}$ is contained in a component of $R_{i}$, then for each $y \in\left\{y_{i-1}, y_{i}\right\}$ and for every component $D$ of $L^{\prime}, N(D) \cap V(C) \subseteq c C y$ or $N(D) \cap V(C) \subseteq y C c^{\prime}$.

Assume that $\left\{x_{i-1}, v_{i-1}\right\} \cup\left\{x_{i}, v_{i}\right\}$ is contained in a component of $R_{i}$. Because $\left(R_{i},\left(x_{i-1}, v_{i-1}, y_{i-1}\right),\left(x_{i}, v_{i}, y_{i}\right), A_{i}, C_{i}\right)$ is a good rung, it follows from (1) or (2) of (2.3) that, for each $x \in\left\{x_{i-1}, x_{i}\right\}, R_{i}-((A-x) \cup C)$ contains a path from $x$ to $\left\{v_{i-1}, v_{i}\right\}$. Hence, (1) follows from (b) (with $x$ as $q \in V(A)$ ). Similarly, we can prove (2).

Claim 4. If $R_{i}$ is connected then $v_{i-1}=v_{i}$.

Suppose $R_{i}$ is connected and $v_{i-1} \neq v_{i}$. Since $\left(R_{i},\left(x_{i-1}, v_{i-1}, y_{i-1}\right),\left(x_{i}, v_{i}, y_{i}\right), A_{i}, C_{i}\right)$ is a good rung, it follows from (1) of (2.3) that $R_{i}-\left(\left(A_{i}-x_{i}\right) \cup\left(C_{i}-y_{i-1}\right)\right)$ contains a $\left(\left\{v_{i-1}, x_{i}\right\},\left\{y_{i-1}, v_{i}\right\}\right)$-linkage $X$ and $R_{i}-\left(\left(A_{i}-x_{i-1}\right) \cup\left(C_{i}-y_{i}\right)\right)$ contains a $\left(\left\{v_{i-1}, y_{i}\right\},\left\{x_{i-1}, v_{i}\right\}\right)$-linkage $Y$.

Then $L^{*}$ has no path from $a A x_{i}-x_{i}$ to $y_{i-1} C c^{\prime}-y_{i-1}$ internally disjoint from $L$. Otherwise, let $P$ be a path from $p \in V\left(a A x_{i}-x_{i}\right)$ to $p^{\prime} \in V\left(y_{i-1} C c^{\prime}-y_{i-1}\right)$ internally disjoint from $L$. Let $K=a A p \cup P \cup p^{\prime} C c^{\prime} \cup c C y_{i-1} \cup x_{i} A a^{\prime} \cup X$. Then, $K \subseteq L_{i, i}$, and $K$ contains disjoint paths from $a, v_{i-1}, c$ to $c^{\prime}, a^{\prime}, v_{i}$, respectively, contradicting (a).

Similarly, we can use $Y$ (instead of $X$ ) to show that $L^{*}$ has no path from $x_{i-1} A a^{\prime}-x_{i-1}$ to $c C y_{i}-y_{i}$ internally disjoint from $L$.

Therefore, $\left\{x_{i-1}, v_{i-1}, y_{i-1}\right\}=\{a, b, c\}$; for otherwise, by Claim $3, L^{*}$ has a separation $\left(G_{1}, G_{2}\right)$ such that $V\left(G_{1} \cap G_{2}\right)=\left\{x_{i-1}, v_{i-1}, y_{i-1}\right\},\{a, b, c\} \subseteq G_{1}$, and $\left\{a^{\prime}, b^{\prime}, c^{\prime}\right\} \subseteq G_{2}$, contradicting Claim 1. Similarly, we can prove $\left\{x_{i}, v_{i}, y_{i}\right\}=\left\{a^{\prime}, b^{\prime}, c^{\prime}\right\}$. Hence by Claim $2, b b^{\prime}$ is the reduced sequence of $v_{0} \ldots v_{m}$.

If $L^{\prime}=\emptyset$, then $L^{*}=R_{i}$, and so, $\left(L^{*},(a, b, c),\left(a^{\prime}, b^{\prime}, c^{\prime}\right)\right)$ is a ladder along $b b^{\prime}$, contradicting the choice of $L^{*}$.

So assume that $L^{\prime} \neq \emptyset$. Let $D$ be a component of $L^{\prime}$. By (f), $|N(D) \cap V(A)| \geq 2$ or $|N(D) \cap V(C)| \geq 2$. By symmetry, let $u, v \in V(A) \cap N(D)$ with $u \neq v$. By (b), $L$ has no path from $V(u A v-\{u, v\})$ to $\left\{b, b^{\prime}\right\}$ internally disjoint from $A \cup C$. Let $u^{\prime}, v^{\prime} \in V(A)$ with $u^{\prime} A v^{\prime}$ maximal such that $u A v \subseteq u^{\prime} A v^{\prime}$ and $L$ has no path from $V\left(u^{\prime} A v^{\prime}-\left\{u^{\prime}, v^{\prime}\right\}\right)$ to $\left\{b, b^{\prime}\right\}$ internally disjoint from $A \cup C$. Then for any component $D^{*}$ of $L^{\prime}, N\left(D^{*}\right) \subseteq u^{\prime} A v^{\prime}$ or $N\left(D^{*}\right) \cap V\left(u^{\prime} A v^{\prime}-\left\{u^{\prime}, v^{\prime}\right\}\right)=\emptyset$. (For otherwise, let $u^{*}, v^{*} \in N\left(D^{*}\right) \cap V(A)$ such that $a, u^{*}, u^{\prime}, v^{*}, v^{\prime}, a^{\prime}$ occur on $A$ in this order and $u^{*}, v^{*} \notin\left\{u^{\prime}, v^{\prime}\right\}$. Then $L$ has no path from $V\left(u^{*} A v^{\prime}-\left\{u^{*}, v^{\prime}\right\}\right)$ to $\left\{b, b^{\prime}\right\}$ internally disjoint from $A \cup C$, and so, $\left\{u^{*}, v^{\prime}\right\}$ contradicts the choice of $\left\{u^{\prime}, v^{\prime}\right\}$.) Also, $L^{*}$ has no path from $V\left(u^{\prime} A v^{\prime}-\left\{u^{\prime}, v^{\prime}\right\}\right)$ to $V(C)$ internally disjoint from $L$; otherwise, by Claim 2, such a path would be from 
$a A x_{i}-x_{i}$ to $y_{i-1} C c^{\prime}-y_{i-1}$ or from $c C y_{i}-y_{i}$ to $x_{i-1} A a^{\prime}-x_{i-1}$, and internally disjoint from $L$, a contradiction. Hence $G-\left\{u^{\prime}, v^{\prime}\right\}$ has a component containing no vertex of $V(A \cup C) \cup\left\{v_{0}, \ldots, v_{m}\right\}$, contradicting (f).

Claim 5. No $R_{i}$ is connected.

Suppose that $R_{i}$ is connected. By Claim $4, v_{i-1}=v_{i}$. By Claim $2, v_{i-1} \neq b$ or $v_{i} \neq b^{\prime}$. By symmetry, assume that $v_{i-1} \neq b$.

If $L^{*}$ has no path from $a A x_{i-1}-x_{i-1}$ to $y_{i-1} C c^{\prime}-y_{i-1}$ internally disjoint from $L$, and $L^{*}$ has no path from $c C y_{i-1}-y_{i-1}$ to $x_{i-1} A a^{\prime}-x_{i-1}$ internally disjoint from $L$, then by Claim $3, L^{*}$ has a separation $\left(G_{1}, G_{2}\right)$ such that $V\left(G_{1} \cap G_{2}\right)=\left\{x_{i-1}, v_{i-1}, y_{i-1}\right\}$, $\{a, b, c\} \subseteq G_{1}$, and $\left\{a^{\prime}, b^{\prime}, c^{\prime}\right\} \subseteq G_{2}$, contradicting Claim 1 .

So assume (by the symmetry between $x_{i-1}$ and $y_{i-1}$ ) that $L^{*}$ has a path $S$ from $s \in V\left(a A x_{i-1}-x_{i-1}\right)$ to $s^{\prime} \in V\left(y_{i-1} C c^{\prime}-y_{i-1}\right)$ internally disjoint from $L$. Select $S$ so that $a A s$ is minimal. By $\left(1^{\prime}\right)$ of $(2.3), R_{i}$ has a path from $y_{i-1}$ to $v_{i}$ internally disjoint from $A \cup C$. Hence for all $j<i, N\left(R_{j}-\left(A_{j} \cup C_{j} \cup\left\{v_{i-1}\right\}\right)\right) \cap V\left(s A x_{i-1}-s\right)=\emptyset$; otherwise, $L_{j, i}$ has disjoint paths from $a, v_{j-1}, c$ to $c^{\prime}, a^{\prime}, v_{i}$, respectively, contradicting (a). Also, by (c) and by Claim $3, L^{*}$ has no path from $c C y_{i-1}-y_{i-1}$ to $s A a^{\prime}-s$ internally disjoint from $L$.

If $N(L-(A \cup C)) \cap a A s=\emptyset$ and $L^{*}$ has no path from $a A s$ to $c C y_{i-1}-y_{i-1}$ internally disjoint from $L$, then by Claim $3, L^{*}$ has a separation $\left(G_{1}, G_{2}\right)$ such that $V\left(G_{1} \cap G_{2}\right)=$ $\left\{a, v_{i-1}, y_{i-1}\right\},\{a, b, c\} \subseteq G_{1}$ and $\left\{a^{\prime}, b^{\prime}, c^{\prime}\right\} \subseteq G_{2}$, contradicting Claim 1 .

So let $r \in V(a A s)$ with $r A s$ minimal such that $r \in N(L-(A \cup C))$ or $L^{*}$ has a path from $r$ to $c C y_{i-1}-y_{i-1}$ internally disjoint from $L$.

If $r \in N\left(L-(A \cup C)\right.$ ), then let $x_{i-1}^{\prime}=r$. By (b) (with $r$ as $q \in V(A)$ there), for every component $D$ of $L^{\prime}, N(D) \cap V(A) \subseteq a A x_{i-1}^{\prime}$ or $N(D) \cap V(A) \subseteq x_{i-1}^{\prime} A a^{\prime}$. If $L^{*}$ has a path from $r$ to $r^{\prime} \in V\left(c C y_{i-1}-y_{i-1}\right)$, then by (d) (with $r, r^{\prime}, s, s^{\prime}$ as $s, s^{\prime}, t, t^{\prime}$, respectively), there is a vertex $x_{i-1}^{\prime} \in r A s$ such that, for every component $D$ of $L^{\prime}$, $N(D) \cap V(A) \subseteq a A x_{i-1}^{\prime}$ or $N(D) \cap V(A) \subseteq x_{i-1}^{\prime} A a^{\prime}$.

Hence by the choices of $r$ and $s$ and by Claim $3, L^{*}$ has a separation $\left(G_{1}, G_{2}\right)$ such that $V\left(G_{1} \cap G_{2}\right)=\left\{x_{i-1}^{\prime}, v_{i-1}, y_{i-1}\right\},\{a, b, c\} \subseteq G_{1}$, and $\left\{a^{\prime}, b^{\prime}, c^{\prime}\right\} \subseteq G_{2}$, contradicting Claim 1.

Claim 6. $A_{i}, C_{i}$ and $\left\{v_{i-1}, v_{i}\right\}$ are contained in three different components of $R_{i}$.

Suppose on the contrary that Claim 6 fails. By Claim 4 and by symmetry, assume that $R_{i}$ is not connected, but $\left\{x_{i-1}, v_{i-1}\right\} \cup\left\{x_{i}, v_{i}\right\}$ is contained in a component of $R_{i}$. Then $y_{i-1}=y_{i}$ (since $\left(R_{i},\left\{x_{i-1}, y_{i-1}\right\},\left\{x_{i}, y_{i}\right\},\left(v_{i-1}, v_{i}\right)\right)$ is a rung). By (e), $v_{i-1}=v_{i}$. Hence, $x_{i-1} \neq x_{i}$, and so, we have $x_{i-1} \neq a^{\prime}$ and $x_{i} \neq a$. Moreover, either $\left\{x_{i-1}, v_{i-1}\right\} \neq\{a, b\}$ or $\left\{x_{i}, v_{i}\right\} \neq\left\{a^{\prime}, b^{\prime}\right\}$; otherwise, $b=v_{i-1}=v_{i}=b^{\prime}$, contradicting Claim 2. By symmetry, assume that $\{a, b\} \neq\left\{x_{i-1}, v_{i-1}\right\} \neq\left\{a^{\prime}, b^{\prime}\right\}$.

If $i=1$ then define $B=\left\{v_{0}\right\}$, and if $i \geq 2$ then define $B=\bigcup_{1 \leq k \leq i-1}\left(R_{k}-\left(A_{k} \cup C_{k}\right)\right)$. Let $B^{\prime}=\bigcup_{k=i}^{m}\left(R_{k}-\left(A_{k} \cup C_{k}\right)\right)$. Then $B \cap B^{\prime}=\left\{v_{i-1}\right\}, N(B) \subseteq a A x_{i-1} \cup c C y_{i-1}$, and 
$N\left(B^{\prime}\right) \subseteq x_{i-1} A a^{\prime} \cup y_{i-1} C c^{\prime}$.

Define $s \in V(C)$ with $s C c^{\prime}$ minimal such that $s=c$, or $s \in N\left(B-v_{i-1}\right)$, or $L^{*}$ has a path from $a A x_{i-1}-x_{i-1}$ to $s$ internally disjoint from $L$. Define $t \in V(C)$ with $c C t$ minimal such that $t=c^{\prime}$, or $t \in N\left(B^{\prime}-v_{i-1}\right)$, or $L^{*}$ contains a path from $x_{i-1} A a^{\prime}-x_{i-1}$ to $t$ internally disjoint from $L$. We distinguish three cases. Note that by (2) of (2.3), $R_{i}-\left(\left(A_{i}-x_{i-1}\right) \cup C_{i}\right)$ has a path from $x_{i-1}$ to $v_{i}$.

Case 1. $s=c$ or $t=c^{\prime}$.

Suppose $s=c$ (respectively, $t=c^{\prime}$ ). Then by Claim 3 and by the choice of $s$ (respectively, $t), L^{*}$ has a separation $\left(G_{1}, G_{2}\right)$ such that $V\left(G_{1} \cap G_{2}\right)=\left\{x_{i-1}, v_{i-1}, c\right\}$ (respectively, $\left.V\left(G_{1} \cap G_{2}\right)=\left\{x_{i-1}, v_{i-1}, c^{\prime}\right\}\right),\{a, b, c\} \subseteq G_{1}$, and $\left\{a^{\prime}, b^{\prime}, c^{\prime}\right\} \subseteq G_{2}$, contradicting Claim 1.

Case 2. $t \neq c^{\prime}$ and $s \in N\left(B-v_{i-1}\right)$.

Then $N\left(B^{\prime}\right) \cap V(c C s-s)=\emptyset$ because $N\left(B^{\prime}\right) \cap V(C) \subseteq y_{i-1} C c^{\prime}$ and $N(B) \cap V(C) \subseteq$ $c C y_{i-1}$. By (b) (with $s$ as $q \in V(C)$ ), for every component $D$ of $L^{\prime}, N(D) \cap V(C) \subseteq c C s$ or $N(D) \cap V(C) \subseteq s C c^{\prime}$. Let $s \in N\left(R_{l}-\left(A_{l} \cup C_{l} \cup\left\{v_{l}\right\}\right)\right)$ for some $l \leq i-1$.

Hence, $L^{*}$ does not contain any path from $x_{i-1} A a^{\prime}-x_{i-1}$ to $c C s-s$; otherwise, $L_{l, i}$ contains three disjoint paths from $a, v_{l-1}, c$ to $v_{i-1}=v_{i}, c^{\prime}, a^{\prime}$, respectively, contradicting (a).

By the choice of $s$ and by Claim $3, L^{*}$ has a separation $\left(G_{1}, G_{2}\right)$ such that $V\left(G_{1} \cap\right.$ $\left.G_{2}\right)=\left\{x_{i-1}, v_{i-1}, s\right\},\{a, b, c\} \subseteq G_{1}$, and $\left\{a^{\prime}, b^{\prime}, c^{\prime}\right\} \subseteq G_{2}$, contradicting Claim 1.

Case 3. $t \neq c^{\prime}$ and $L^{*}$ has a path $S$ from $a A x_{i-1}-x_{i-1}$ to $s$ internally disjoint from $L$.

If $L^{*}$ has a path from $x_{i-1} A a^{\prime}-x_{i-1}$ to $t$ internally disjoint from $L$, then $t \in s C c^{\prime}$ by (c) (with $s, t$ as $t, t^{\prime}$ in (c), respectively). By (d) (with $s, t$ as $t, t^{\prime}$ in (d), respectively), there is some vertex $q \in s C t$ such that, for every component $D$ of $L^{\prime}, N(D) \cap V(C) \subseteq c C q$ or $N(D) \cap V(C) \subseteq q C c^{\prime}$. So by Claim 3 and by the choices of $s$ and $t, L^{*}$ has a separation $\left(G_{1}, G_{2}\right)$ such that $V\left(G_{1} \cap G_{2}\right)=\left\{x_{i-1}, v_{i-1}, q\right\},\{a, b, c\} \subseteq G_{1}$, and $\left\{a^{\prime}, b^{\prime}, c^{\prime}\right\} \subseteq G_{2}$, contradicting Claim 1.

Now assume that $t \in N\left(B^{\prime}-v_{i-1}\right)$. Then by (b) (with $t$ as $q \in V(C)$ ), for every component $D$ of $L^{\prime}, N(D) \cap V(C) \subseteq c C t$ or $N(D) \cap V(C) \subseteq t C c^{\prime}$. Let $t \in N\left(R_{l}-\left(A_{l} \cup C_{l} \cup\right.\right.$ $\left.\left.\left\{v_{l-1}\right\}\right)\right)$ for some $l$ with $i \leq l \leq m$. Hence, $t \in s C c^{\prime}$; otherwise, $L_{i, l}$ contains three disjoint paths from $a, v_{i-1}, c$ to $c^{\prime}, a^{\prime}, v_{l}$, respectively, contradicting (a). Thus, by Claim 3 and by the choices of $s$ and $t, L^{*}$ has a separation $\left(G_{1}, G_{2}\right)$ such that $V\left(G_{1} \cap G_{2}\right)=\left\{x_{i-1}, v_{i-1}, t\right\}$, $\{a, b, c\} \subseteq G_{1}$, and $\left\{a^{\prime}, b^{\prime}, c^{\prime}\right\} \subseteq G_{2}$, contradicting Claim 1. This proves Claim 6 .

By Claim 6, $A, C$ and $\left\{v_{0}, \ldots, v_{m}\right\}$ are in different components of $L$. Hence, $A \cup C$ and $\left\{b, b^{\prime}\right\}$ are contained in different components of $L^{*}$. Since $b \neq b^{\prime}$ (by Claim 2), $G$ has a separation $\left(G_{1}, G_{2}\right)$ such that $V\left(G_{1} \cap G_{2}\right)=\left\{a, b^{\prime}, c\right\},\{a, b, c\} \subseteq G_{1}$, and $\left\{a^{\prime}, b^{\prime}, c^{\prime}\right\} \subseteq G_{2}$, contradicting Claim 1. 


\section{The proof of main result}

First, we prove the following lemma, which proves the necessary part of (1.3).

(3.1) Lemma. Let $\left(G,\{a, c\},\left\{a^{\prime}, c^{\prime}\right\},\left(b, b^{\prime}\right)\right)$ be an obstruction. Then one of the following statements holds.

(1) $G$ has a separation $\left(G_{1}, G_{2}\right)$ of order at most 2 such that $\{a, b, c\} \subseteq V\left(G_{1}\right)$ and $\left\{a^{\prime}, b^{\prime}, c^{\prime}\right\} \subseteq V\left(G_{2}\right)$.

(2) There is a subset $T \subseteq V(G)$ such that $|T| \leq 3$ and some component of $G-T$ contains no element of $\{a, b, c\} \cup\left\{a^{\prime}, b^{\prime}, c^{\prime}\right\}$.

(3) $\left(G,(a, b, c),\left(a^{\prime}, b^{\prime}, c^{\prime}\right)\right)$ is a ladder.

(4) $G$ has a separation $(J, L)$ such that $V(J \cap L)=\left\{w_{0}, \ldots, w_{n}\right\},\left(J, w_{0}, \ldots, w_{n}\right)$ is 3-planar, $\left(L,(a, b, c),\left(a^{\prime}, b^{\prime}, c^{\prime}\right)\right)$ is a ladder along a sequence $v_{0} \ldots v_{m}$, where $v_{0}=b$, $v_{m}=b^{\prime}$, and $w_{0} \ldots w_{n}$ is the reduced sequence of $v_{0} \ldots v_{m}$.

Proof. Assume that (1) and (2) do not hold. Let $A, B, C$ be disjoint paths from $\{a, b, c\}$ to $\left\{a^{\prime}, b^{\prime}, c^{\prime}\right\}$ in $G$ such that $a \in A, c \in C$, and $B$ is from $b$ to $b^{\prime}$. We choose $A, B$ and $C$ to be induced paths. Let $H^{\prime}$ denote the component of $G-(A \cup C)$ containing $B$. Let $K$ be the subgraph of $G$ obtained from $A \cup C \cup H^{\prime}$ by adding those edges in $G$ between $V(A) \cup V(C)$ and $V\left(H^{\prime}\right)$. It is straightforward to verify that $A, B, C, H^{\prime}$ and $K$ satisfy the conditions (i)-(iii) of (2.4) (with $K$ as $G$ there). By applying (2.4), one of (1)-(4) of (2.4) holds.

Because of $A, B, C,(1)$ of (2.4) does not hold. Since we assume that (2) of (3.1) does not hold, if there is some $T \subseteq V(K)$ with $|T| \leq 3$ such that some component $U$ of $G-T$ contains no element of $\{a, b, c\} \cup\left\{a^{\prime}, b^{\prime}, c^{\prime}\right\}$, then $T$ would be contained in the "ladder part" of $K$ when (3) or (4) of (2.4) holds (see the comment after (1.3)). So we may assume that either (1a) $\left(K,(a, b, c),\left(a^{\prime}, b^{\prime}, c^{\prime}\right), A, C\right)$ (when $a^{\prime} \in A$ and $c^{\prime} \in C$ ) or $\left(K,(a, b, c),\left(c^{\prime}, b^{\prime}, a^{\prime}\right), A, C\right)$ (when $a^{\prime} \in C$ and $c^{\prime} \in A$ ) is a good ladder along a sequence $v_{0} \ldots v_{m}$, where $v_{0}=b$ and $v_{m}=b^{\prime}$ (in this case, let $J=\left\{w_{0}, \ldots, w_{n}\right\}$ and $L=K$, where $w_{0} \ldots w_{n}$ is the reduced sequence of $\left.v_{0} \ldots v_{m}\right)$, or (1b) $K$ has a separation $(J, L)$ such that $V(J \cap L)=\left\{w_{0}, \ldots, w_{n}\right\},\left(J, w_{0}, \ldots, w_{n}\right)$ is 3-planar, $\left(L,(a, b, c),\left(a^{\prime}, b^{\prime}, c^{\prime}\right), A, C\right)$ (when $a^{\prime} \in A$ and $c^{\prime} \in C$ ) or $\left(L,(a, b, c),\left(c^{\prime}, b^{\prime}, a^{\prime}\right), A, C\right)$ (when $a^{\prime} \in C$ and $c^{\prime} \in A$ ) is a good ladder along $v_{0} \ldots v_{m}$, where $v_{0}=b, v_{m}=b^{\prime}$, and $w_{0} \ldots w_{n}$ is the reduced sequence of $v_{0} \ldots v_{m}$.

Let $\left(R_{i},\left(x_{i-1}, v_{i-1}, y_{i-1}\right),\left(x_{i}, v_{i}, y_{i}\right), A_{i}, C_{i}\right), i=1, \ldots, m$, be the good rungs of $L$, where $A_{i}=A \cap R_{i}, C_{i}=C \cap R_{i}, a=x_{0}, c=y_{0}, a^{\prime}=x_{m}$ (when $a^{\prime} \in A$ ) or $c^{\prime}=x_{m}$ (when $c^{\prime} \in A$ ), and $c^{\prime}=y_{m}\left(\right.$ when $c^{\prime} \in C$ ) or $a^{\prime}=y_{m}$ (when $\left.a^{\prime} \in C\right)$. Let $v(L)=\{x \in$ $\left.V\left(R_{k}-v_{k}\right): v_{k-1}=v_{k}, 1 \leq k \leq m\right\}$. We select $A, B, C, H^{\prime}, J$ and $L$ such that

(I) $H^{\prime}$ is maximal, 
(II) subject to (I), $v(L)$ is maximal, and

(III) subject to (II), $J$ is maximal.

Without loss of generality, we may choose the notation so that $A, B, C$ are from $a, b, c$ to $a^{\prime}, b^{\prime}, c^{\prime}$, respectively. Let $L^{\prime}=G-K$ and let $L^{*}=G\left[V\left(L \cup L^{\prime}\right)\right]$. Then $L^{*}$ is obtained from the disjoint union of $L$ and $L^{\prime}$ by adding edges between $V(A) \cup V(C)$ and $V\left(L^{\prime}\right)$ or between $V(A)$ and $V(C)$. Next we will show that $L^{*}, L, L^{\prime}, A, C$ satisfy the remaining conditions (a)-(f) of (2.5).

Note that since (2) does not hold for $G, J$ can be drawn in a closed disc in the plane with no edge crossings such that $w_{0}, w_{1}, \ldots, w_{n}$ occur on the boundary of the disc in that cyclic order.

(a) For any $i, j \in\{1, \ldots, m\}$ with $i \leq j$, let $L_{i, j}$ denote the graph obtained from $L^{*}$ by deleting all $R_{k}-\left(A_{k} \cup C_{k} \cup\left\{v_{i-1}, v_{j}\right\}\right)$ with $k<i$ or $k>j$ and by deleting $v_{0}, \ldots, v_{m}$ except $v_{i-1}$ and $v_{j}$. Then $\left(L_{i, j},\{a, c\},\left\{a^{\prime}, c^{\prime}\right\},\left(v_{i-1}, v_{j}\right)\right)$ is an obstruction.

Clearly (a) holds when $v_{i-1}=v_{j}$. So assume that $v_{i-1} \neq v_{j}$. For convenience, let $G^{*}$ denote the union of $J$ and all $R_{k}-\left(A_{k} \cup C_{k}\right)$ with $k<i$ or $k>j$. Then $V\left(G^{*} \cap L_{i, j}\right)=\left\{v_{i-1}, v_{j}\right\}$.

If we let $\mathcal{J}=\left\{V\left(R_{k}\right)-\left(V\left(A_{k} \cup C_{k}\right) \cup\left\{v_{k-1}, v_{k}\right\}\right): k<i\right.$ or $k>$ $j\}$, then $\left(G^{*}, \mathcal{J}, v_{0}, \ldots, v_{i-1}, v_{j}, \ldots, v_{m}\right)$ is 3 -planar. Hence, let $\mathcal{J}^{*}$ be a collection of pairwise disjoint subsets of $V(J)-\left\{v_{0}, \ldots, v_{i-1}, v_{j}, \ldots, v_{m}\right\}$ such that $\left(G^{*}, \mathcal{J}^{*}, v_{0}, \ldots, v_{i-1}, v_{j}, \ldots, v_{m}\right)$ is 3-planar and $\mathcal{J}^{*}$ is minimal.

If $G^{*}$ contains a $\left(\left\{b, v_{i-1}\right\},\left\{b^{\prime}, v_{j}\right\}\right)$-linkage, then $\left(L_{i, j},\{a, c\},\left\{a^{\prime}, c^{\prime}\right\},\left(v_{i-1}, v_{j}\right)\right)$ is an obstruction. For otherwise, $L_{i, j}$ has three disjoint paths form $\left\{a, v_{i-1}, c\right\}$ to $\left\{a^{\prime}, v_{j}, c^{\prime}\right\}$, none from $v_{i-1}$ to $v_{j}$. These three paths in $L_{i, j}$ combined with a $\left(\left\{b, v_{i-1}\right\},\left\{b^{\prime}, v_{j}\right\}\right)$ linkage in $G^{*}$ give three disjoint paths in $G$ from $\{a, b, c\}$ to $\left\{a^{\prime}, b^{\prime}, c^{\prime}\right\}$, none from $b$ to $b^{\prime}$. This contradicts the assumption that $\left(G,\{a, c\},\left\{a^{\prime}, c^{\prime}\right\},\left(b, b^{\prime}\right)\right)$ is an obstruction.

So assume that $G^{*}$ contains no $\left(\left\{b, v_{i-1}\right\},\left\{b^{\prime}, v_{j}\right\}\right)$-linkage. Then $p\left(G^{*}, \mathcal{J}^{*}\right)$ has no $\left(\left\{b, v_{i-1}\right\},\left\{b^{\prime}, v_{j}\right\}\right)$-linkage; otherwise, by $(2.2)$ (with $G^{*}, \mathcal{J}^{*}, v_{i-1}, v_{j}$ as $G, \mathcal{A}, u, v$, respectively), $G^{*}$ would contain a $\left(\left\{b, v_{i-1}\right\},\left\{b^{\prime}, v_{j}\right\}\right)$-linkage. By planarity, $p\left(G^{*}, \mathcal{J}^{*}\right)$ has a separation $\left(G_{1}^{\prime}, G_{2}^{\prime}\right)$ such that $\left|V\left(G_{1}^{\prime} \cap G_{2}^{\prime}\right)\right| \leq 1,\left\{v_{i-1}, v_{j}\right\} \subseteq G_{1}^{\prime}$, and $\left\{b, b^{\prime}\right\} \subseteq G_{2}^{\prime}$. Again by planarity of $p\left(G^{*}, \mathcal{J}^{*}\right)$, there are $r \leq i-1$ and $s \geq j$ such that $\left\{v_{r}, \ldots, v_{s}\right\} \subseteq G_{1}^{\prime}$ and $\left\{v_{0}, \ldots, v_{r-1}, v_{s+1}, \ldots, v_{m}\right\} \subseteq G_{2}^{\prime}$. Let $G_{i}$ denote the subgraph of $G^{*}$ induced by the vertices contained in $V\left(G_{i}^{\prime}\right)$ or contained in some $U \in \mathcal{J}^{*}$ with $N_{G^{*}}(U) \subseteq V\left(G_{i}^{\prime}\right)$. Then $\left(G_{1}, G_{2}\right)$ is a separation of $G^{*}$ such that $\left|V\left(G_{1} \cap G_{2}\right)\right| \leq 1,\left\{v_{r}, \ldots, v_{s}\right\} \subseteq G_{1}$, and $\left\{v_{0}, \ldots, v_{r-1}, v_{s+1}, \ldots, v_{m}\right\} \subseteq G_{2}$.

Because $K-(A \cup C)$ is connected, $\left|V\left(G_{1} \cap G_{2}\right)\right|=1$. Let $w$ be the vertex in $V\left(G_{1} \cap G_{2}\right)$. Then $K$ has a separation $\left(M_{1}, M_{2}\right)$ such that $V\left(M_{1} \cap M_{2}\right)=\left\{x_{r-1}, y_{r-1}, w, x_{s}, y_{s}\right\}$, $M_{1}=G_{1} \cup\left(\bigcup_{r \leq k \leq s} R_{k}\right)$, and $G_{2} \subseteq M_{2}$. 
Let $a_{0}, \ldots, a_{l}$ be vertices in that order on $A$ and $c_{0}, \ldots, c_{l}$ be vertices in that order on $C$ such that (a1) $a_{0}=x_{r-1}, a_{l}=x_{s}, c_{0}=y_{r-1}$, and $c_{l}=y_{s}$, (a2) for $i=1, \ldots, l-1$, $\left\{a_{i}, c_{i}\right\}$ is a 2-cut of $M_{1}-w$, and (a3) for any $x \in V\left(a_{i-1} A a_{i}\right)$ and $y \in V\left(c_{i-1} C c_{i}\right),\{x, y\}$ is not a 2-cut of $M_{1}-w$ unless $\{x, y\}=\left\{a_{i-1}, c_{i-1}\right\}$ or $\{x, y\}=\left\{a_{i}, c_{i}\right\}$. Let $S$ denote the set of edges of $M_{1}$ with both ends contained in $\left\{a_{k}, c_{k}, w\right\}, k=0, \ldots, l$.

Let $M_{1}^{k}$ denote the subgraph of $M_{1}-S$ induced by those vertices $x$ such that every path in $M_{1}-w$ from $x$ to $\left\{x_{r-1}, y_{r-1}, w, x_{s}, y_{s}\right\}$ intersect $\left\{a_{k-1}, a_{k}, w, c_{k-1}, c_{k}\right\}$. Because (2) does not hold for $G$ and by (a3), $M_{1}^{k}$ has no 3 -separation $\left(M^{\prime}, M^{\prime \prime}\right)$ such that $\left\{a_{k-1}, w, c_{k-1}\right\} \subseteq M^{\prime}$ and $\left\{a_{k}, w, c_{k}\right\} \subseteq M^{\prime \prime}$. By (1) of (1.1), $\left(M_{1}^{k},\left(a_{k-1}, w, c_{k-1}\right),\left(a_{k}, w, c_{k}\right)\right)$ is a rung. In fact, by (a3), it is easy to show that $\left(M_{1}^{k},\left(a_{k-1}, w, c_{k-1}\right),\left(a_{k}, w, c_{k}\right), a_{k-1} A a_{k}, c_{k-1} C c_{k}\right)$ is a good rung.

Again because (2) does not hold for $G$ and by (a3), $M_{1}=\left(\bigcup_{k=1}^{l} M_{1}^{k}\right)+S$. Therefore, we see that $\left(M_{1},\left(x_{r-1}, v_{r}, y_{r-1}\right),\left(x_{s}, v_{s}, y_{s}\right), A \cap M_{1}, C \cap M_{1}\right)$ is a good ladder along $w$.

Let $L_{1}=M_{1} \cup L$, and let $J_{1}=J-\left(G_{1}-w\right)$. Then $\left(J_{1}, L_{1}\right)$ is a separation of $K$ such that $V\left(J_{1} \cap L_{1}\right)=\left\{w_{0}, \ldots, w_{t-1}, w, w_{t}, \ldots, w_{n}\right\}$ for some appropriate $t$, $\left(J_{1}, w_{0}, \ldots, w_{t-1}, w, w_{t}, \ldots, w_{n}\right)$ is 3-planar, $\left(L_{1},(a, b, c),\left(a^{\prime}, b^{\prime}, c^{\prime}\right), A, C\right)$ is a good ladder along a sequence whose reduced sequence is $w_{0} \ldots w_{t-1} w w_{t} \ldots w_{n}$. Note that $H^{\prime}$ remains unchanged, but $v(L) \cup\left(\left\{v_{i-1}, v_{j}\right\}-\{w\}\right) \subseteq v\left(L_{1}\right)$, and $v_{i-1}, v_{j} \notin v(L)$, contradicting (II).

(b) If $q \in V(A)$ (respectively, $q \in V(C)$ ) such that $L$ contains a path from $q$ to $\left\{v_{0}, \ldots, v_{m}\right\}$ internally disjoint from $V(A \cup C) \cup\left\{v_{0}, \ldots, v_{m}\right\}$, then for every component $D$ of $L^{\prime}, N(D) \cap V(A) \subseteq a A q$ or $N(D) \cap V(A) \subseteq q A a^{\prime}$ (respectively, $N(D) \cap V(C) \subseteq c C q$ or $\left.N(D) \cap V(C) \subseteq q C c^{\prime}\right)$.

Suppose (b) is false. By symmetry, assume that $L$ contains a path from $q \in V(A)$ to $\left\{v_{0}, \ldots, v_{m}\right\}$ internally disjoint from $V(A \cup C) \cup\left\{v_{0}, \ldots, v_{m}\right\}$, and assume that there is a component $D$ of $L^{\prime}$ such that $N(D) \cap V(a A q-q) \neq \emptyset$ and $N(D) \cap V\left(q A a^{\prime}-q\right) \neq \emptyset$. Let $u \in N(D) \cap V(a A q-q)$ and $v \in N(D) \cap V\left(q A a^{\prime}-q\right)$.

Let $A^{\prime}$ be an induced path from $a$ to $a^{\prime}$ in $L^{*}$ such that $V\left(A^{\prime}\right) \subseteq a A u \cup D \cup v A a^{\prime}$. Then the component of $G-\left(A^{\prime} \cup C\right)$ containing $B$ is larger than $H^{\prime}$, contradicting (I).

(c) If $L^{*}$ contains paths from distinct $s, s^{\prime} \in V(A)$ (in that order from $a$ to $a^{\prime}$ ) to distinct $t, t^{\prime} \in V(C)$ (in that order from $c^{\prime}$ to $c$ ), respectively, which are internally disjoint from $L$, then $L$ contains no path from $V\left(s A s^{\prime}-\left\{s, s^{\prime}\right\}\right) \cup V\left(t C t^{\prime}-\left\{t, t^{\prime}\right\}\right)$ to $\left\{v_{0}, \ldots, v_{m}\right\}$ internally disjoint from $V(A \cup C) \cup\left\{v_{0}, \ldots, v_{m}\right\}$.

Let $P, P^{\prime}$ be the paths in (c) from $s, s^{\prime}$ to $t, t^{\prime}$, respectively, which are internally disjoint from $L$. If $P \cap P^{\prime} \neq \emptyset$, then there is a component $D$ of $L^{\prime}$ such that $\left\{s, s^{\prime}, t, t^{\prime}\right\} \subseteq N(D)$, and (c) follows from (b). So assume that $P \cap P^{\prime}=\emptyset$.

Suppose that (c) fails, and assume by symmetry that $L^{*}$ has a path from $V\left(s A s^{\prime}-\right.$ $\left.\left\{s, s^{\prime}\right\}\right)$ to $\left\{v_{0}, \ldots, v_{m}\right\}$ internally disjoint from $V(A \cup C) \cup\left\{v_{0}, \ldots, v_{m}\right\}$. Let $A^{\prime}$ be an 
induced path in $L^{*}$ from $a$ to $c^{\prime}$ such that $V\left(A^{\prime}\right) \subseteq a A s \cup P \cup t C c^{\prime}$, and let $C^{\prime}$ be an induced path in $L^{*}$ from $c$ to $a^{\prime}$ such that $V\left(C^{\prime}\right) \subseteq c C t^{\prime} \cup P^{\prime} \cup s^{\prime} A a^{\prime}$. Then the component of $G-\left(A^{\prime} \cup C^{\prime}\right)$ containing $B$ is larger than $H^{\prime}$, contradicting (I).

(d) Suppose that $L^{*}$ contains paths from $s, s^{\prime} \in V(A)$ (in that order from $a$ to $a^{\prime}$ ) to $t, t^{\prime} \in V(C)$ (in that order from $c$ to $c^{\prime}$ ) which are internally disjoint from $L$. If $L$ contains a path from $V\left(t C t^{\prime}-\left\{t, t^{\prime}\right\}\right)$ (respectively, $V\left(s A s^{\prime}-\left\{s, s^{\prime}\right\}\right)$ ) to $\left\{v_{0}, \ldots, v_{m}\right\}$ internally disjoint from $V(A \cup C) \cup\left\{v_{0}, \ldots, v_{m}\right\}$, then there is a vertex $q \in s A s^{\prime}$ (respectively, $q \in t C t^{\prime}$ ) such that, for every component $D$ of $L^{\prime}$, $N(D) \cap V(A) \subseteq a A q$ or $N(D) \cap V(A) \subseteq q A a^{\prime}$ (respectively, $N(D) \cap V(C) \subseteq c C q$ or $\left.N(D) \cap V(C) \subseteq q C c^{\prime}\right)$.

By symmetry, assume $L$ contains a path from $V\left(t C t^{\prime}-\left\{t, t^{\prime}\right\}\right)$ to $\left\{v_{0}, \ldots, v_{m}\right\}$ internally disjoint from $V(A \cup C) \cup\left\{v_{0}, \ldots, v_{m}\right\}$. Let $r, r^{\prime} \in V(A)$ with $r A r^{\prime} \subseteq s A s^{\prime}$ and $r A r^{\prime}$ minimal such that $L^{*}$ has paths $R, R^{\prime}$ from $r, r^{\prime} \in V(A)$ to $t, t^{\prime}$, respectively, which are internally disjoint from $L$. By (c), $a, s, r, r^{\prime}, s^{\prime}, a^{\prime}$ occur on $A$ in that order. We will show that there is some $q \in V\left(r A r^{\prime}\right)$ such that, for every component $D$ of $L^{\prime}$, $N(D) \cap V(A) \subseteq a A q$ or $N(D) \cap V(A) \subseteq q A a^{\prime}$.

We may assume that $L^{\prime}$ has a component $D_{1}$ such that $N\left(D_{1}\right) \cap V(a A r-r) \neq \emptyset$ and $N\left(D_{1}\right) \cap V\left(r A a^{\prime}-r\right) \neq \emptyset$; otherwise, $q:=r$ would be the desired vertex. Let $p_{1} \in V(a A r-r) \cap N\left(D_{1}\right)$ and $q_{1} \in V\left(r A a^{\prime}-r\right) \cap N\left(D_{1}\right)$, and let $P_{1}$ be a path in $L^{*}$ from $p_{1}$ to $q_{1}$ through $D_{1}$ and internally disjoint from $L$. Select $D_{1}, P_{1}, p_{1}, q_{1}$ so that $q_{1} A a^{\prime}$ is minimal and subject to this, $p_{1} A q_{1}$ is maximal. See Figure 1.

Then $q_{1} \in r A r^{\prime}-r$. For otherwise, $q_{1} \in r^{\prime} A c^{\prime}-r^{\prime}$. By (c), $D_{1} \cap\left(R \cup R^{\prime}\right)=\emptyset$. Let $A^{\prime}$ be an induced path in $L^{*}$ from $a$ to $a^{\prime}$ such that $V\left(A^{\prime}\right) \subseteq a A p_{1} \cup P_{1} \cup q_{1} A a^{\prime}$, and let $C^{\prime}$ be an induced path from $c$ to $c^{\prime}$ in $L^{*}$ such that $V\left(C^{\prime}\right) \subseteq c C t \cup R \cup r A r^{\prime} \cup R^{\prime} \cup t^{\prime} C c^{\prime}$. Then the component of $G-\left(A^{\prime} \cup C^{\prime}\right)$ containing $B$ is larger than $H^{\prime}$, contradicting (I).

By (c), $R^{\prime} \cap D_{1}=\emptyset$. By the minimality of $r A r^{\prime}, R \cap D_{1}=\emptyset$. We may assume that there are $p_{2} \in V\left(a A q_{1}-q_{1}\right)$ and $q_{2} \in V\left(q_{1} A a^{\prime}-q_{1}\right)$ such that $\left\{p_{2}, q_{2}\right\} \subseteq N\left(D_{2}\right)$ for some component $D_{2}$ of $L^{\prime}$; otherwise, $q:=q_{1}$ would be the desired vertex. Let $P_{2}$ be a path in $L^{*}$ from $p_{2}$ to $q_{2}$ through $D_{2}$ and internally disjoint from $L$. Select $D_{2}, P_{2}, p_{2}, q_{2}$ such that $q_{2} A a^{\prime}$ is minimal, and subject to this, $p_{2} A q_{2}$ is maximal. By the choice of $D_{1}, P_{1}, p_{1}$ and $q_{1}$, we conclude that $p_{2} \in r A q_{1}-q_{1}$. If $q_{2} \in r^{\prime} A a^{\prime}-r^{\prime}$, then we stop this process; and if $q_{2} \in q_{1} A r^{\prime}-q_{1}$, then we find $D_{3}, P_{3}, p_{3}, q_{3}$ in a similar way. Since $G$ is a finite graph, we have a sequence $P_{1}, P_{2}, \ldots, P_{l}$ of paths with $P_{i}$ from $p_{i}$ to $q_{i}$ such that $q_{l} \in r^{\prime} A a^{\prime}-r^{\prime}$, and for $i \in\{1, \ldots, l-1\}, p_{i+1} \in q_{i-1} A q_{i}-q_{i}$ and $q_{i} \in p_{i+1} A p_{i+2}-p_{i+1}$, where $q_{0}=r$ and $p_{l+1}=r^{\prime}$. Figure 1 illustrates the cases for $l=3$ and $l=4$.

By (c) and by the minimality of $r A r^{\prime},\left(P_{i}-\left\{p_{i}, q_{i}\right\}\right) \cap\left(R \cup R^{\prime}\right)=\emptyset$ for $i \in\{1, \ldots, l\}$. By the choice of $P_{i}$, all $P_{i}$ 's are internally disjoint, and $P_{i} \cap P_{j}=\emptyset$ if $|i-j| \neq 2$.

If $l$ is odd, then let $A^{\prime}$ be an induced path in $L^{*}$ from $a$ to $a^{\prime}$ such that $V\left(A^{\prime}\right) \subseteq$ $a A p_{1} \cup P_{1} \cup q_{1} A p_{3} \cup P_{3} \cup q_{3} A p_{5} \cup \ldots \cup P_{l} \cup q_{l} A a^{\prime}$, and let $C^{\prime}$ be an induced path in $L^{*}$ from $c$ 


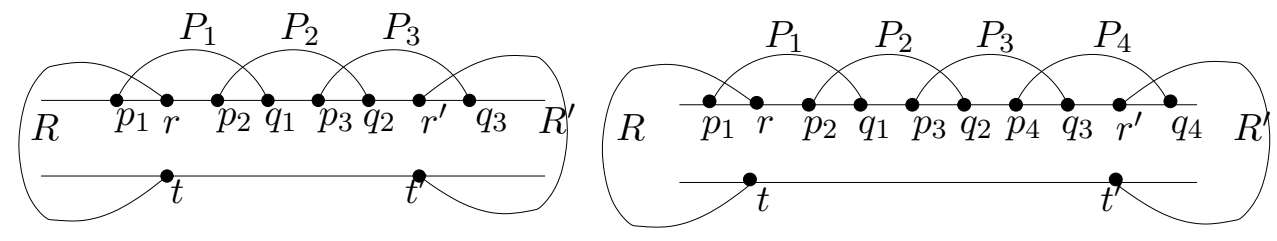

Figure 1: Paths $P_{1}, \ldots, P_{l}$.

to $c^{\prime}$ such that $V\left(C^{\prime}\right) \subseteq c C t \cup R \cup r A p_{2} \cup P_{2} \cup q_{2} A p_{4} \cup P_{4} \cup \ldots \cup P_{l-1} \cup q_{l-1} A r^{\prime} \cup R^{\prime} \cup t^{\prime} C c^{\prime}$. If $l$ is even, then let $A^{\prime}$ be an induced path in $L^{*}$ from $a$ to $c^{\prime}$ such that $V\left(A^{\prime}\right) \subseteq$ $a A p_{1} \cup P_{1} \cup q_{1} A p_{3} \cup P_{3} \cup q_{3} A p_{5} \cup \ldots \cup P_{l-1} \cup q_{l-1} A r^{\prime} \cup R^{\prime} \cup t^{\prime} C c^{\prime}$, and let $C^{\prime}$ be an induced path in $L^{*}$ from $c$ to $a^{\prime}$ such that $V\left(C^{\prime}\right) \subseteq c C t \cup R \cup r A p_{2} \cup P_{2} \cup q_{2} A p_{4} \cup P_{4} \cup \ldots \cup P_{l} \cup q_{l} A a^{\prime}$. Clearly, the component of $G-\left(A^{\prime} \cup C^{\prime}\right)$ containing $B$ is larger than $H^{\prime}$, contradicting (I).

(e) If $R_{i}$ is not connected and $\left\{x_{i-1}, v_{i-1}\right\} \cup\left\{x_{i}, v_{i}\right\}$ (respectively, $\left\{y_{i-1}, v_{i-1}\right\} \cup\left\{y_{i}, v_{i}\right\}$ ) is contained in a component of $R_{i}$, then $v_{i-1}=v_{i}$.

Suppose $v_{i-1} \neq v_{i}$. Assume by symmetry that $\left\{x_{i-1}, v_{i-1}\right\} \cup\left\{x_{i}, v_{i}\right\}$ is contained in a component $U$ of $R_{i}$. Then $y_{i-1}=y_{i}$ and $|V(U)| \geq 4$ because $E(U) \neq \emptyset$ and $R_{i}$ has no 3-separation $\left(R^{\prime}, R^{\prime \prime}\right)$ such that $\left\{x_{i-1}, v_{i-1}, y_{i-1}\right\} \subseteq R^{\prime}$ and $\left\{x_{i}, v_{i}, y_{i}\right\} \subseteq R^{\prime \prime}$. By assumption that (2) does not hold for $G, x_{i-1} \neq x_{i}$;

Moreover, there is a collection $\mathcal{U}$ of pairwise disjoint subsets of $V(U)-$ $\left\{x_{i-1}, v_{i-1}, x_{i}, v_{i}\right\}$ such that $\left(U, \mathcal{U}, x_{i-1}, x_{i}, v_{i}, v_{i-1}\right)$ is 3 -planar; otherwise, $U$ contains an $\left(\left\{x_{i-1}, v_{i}\right\},\left\{v_{i-1}, x_{i}\right\}\right)$-linkage (by (2.1)), and so, $L_{i, i}$ has disjoint paths from $a, v_{i-1}, c$ to $v_{i}, a^{\prime}, c^{\prime}$, respectively, contradicting (a).

Note that $U-A_{i}$ is connected. We claim that $\left\{v_{i-1}, v_{i}\right\}$ is contained in a block $M$ of $U-A_{i}$. For otherwise, let $\left(U_{1}, U_{2}\right)$ be a 1-separation in $U-A_{i}$ such that $v_{i-1} \in U_{1}$ and $v_{i} \in U_{2}$. Then there are $u_{j} \in V\left(A_{i}\right) \cap N\left(U_{j}\right), j=1,2$, such that $x_{i-1}, u_{2}, u_{1}, x_{i}$ occur on $A$ in that order; as otherwise $U$ has a 2-separation $\left(U_{1}^{\prime}, U_{2}^{\prime}\right)$ such that $U_{1} \cup\left\{x_{i-1}\right\} \subseteq U_{1}^{\prime}$ and $U_{2} \cup\left\{x_{i}\right\} \subseteq U_{2}^{\prime}$, and $\left(U_{1}^{\prime} \cup\left\{y_{i}\right\}, U_{2}^{\prime} \cup\left\{y_{i}\right\}\right)$ would be a 3 -separation in $R_{i}$, a contradiction. Hence, $U$ has an $\left(\left\{x_{i-1}, v_{i}\right\},\left\{v_{i-1}, x_{i}\right\}\right)$-linkage. This implies that $L_{i, i}$ has disjoint paths from $a, v_{i-1}, c$ to $v_{i}, a^{\prime}, c^{\prime}$, respectively, contradicting (a).

We claim that $X \cap V(M)=\emptyset$ for all $X \in \mathcal{U}$. Otherwise, let $X \in \mathcal{U}$ such that $X \cap V(M) \neq \emptyset$. Then $\left|N_{U}(X) \cap V(M)\right| \geq 2$ because $M$ is 2-connected. Hence, $\mid N_{U}(X) \cap$ $V(A) \mid \leq 1$. Therefore $G-N_{U}(X)$ has a component containing no element of $\{a, b, c\} \cup$ $\left\{a^{\prime}, b^{\prime}, c^{\prime}\right\}$, contradicting the assumption that (2) does not hold for $G$.

Thus $M$ is a plane subgraph of $p(U, \mathcal{U})$. Let $b_{0}, \ldots, b_{k}$ be the vertices on the outer cycle of $M$ (in that clockwise order from $b_{0}=v_{i-1}$ to $b_{k}=v_{i}$ ) such that, for each $j \in$ $\{1, \ldots, k-1\}, b_{j} \in N(A)$ or $b_{j}$ is a cut vertex of $U-A$. Let $R_{i}^{\prime}=R_{i}-\left(M-\left\{b_{0}, \ldots, b_{k}\right\}\right)$. 
By 3-planarity of $U$, it is easy to verify that $\left(R_{i}^{\prime},\left(x_{i-1}, v_{i-1}, y_{i}\right),\left(x_{i}, v_{i}, y_{i}\right)\right)$ is a ladder along a sequence whose reduced sequence is $b_{0} \ldots b_{k}$. In fact, it is easy to see that $\left(R_{i}^{\prime},\left(x_{i-1}, v_{i-1}, y_{i}\right),\left(x_{i}, v_{i}, y_{i}\right), A_{i}, C_{i}\right)$ is a good ladder (its rungs are not connected).

Let $J_{1}=J \cup M$ and let $L_{1}=L-\left(M-\left\{b_{0}, \ldots, b_{k}\right\}\right)$. Then $\left(J_{1}, L_{1}\right)$ is a separation in $K$ such that $V\left(L_{1} \cap J_{1}\right)=\left\{w_{0}, \ldots, w_{n}\right\} \cup\left\{b_{0}, \ldots, b_{k}\right\}$, $\left(L_{1}, w_{0}, \ldots, w_{j-1}, b_{0}, \ldots, b_{k}, w_{j}, \ldots, w_{n}\right)$ is 3 -planar (for some appropriate $j$ such that $w_{j-1}=v_{r}$ and $w_{j}=v_{s}$ for some $r \leq i-1$ and $\left.s \geq i\right),\left(L_{1},(a, b, c),\left(a^{\prime}, b^{\prime}, c^{\prime}\right), A, C\right)$ is a good ladder along a sequence $S$, where $w_{0} \ldots w_{j-1} b_{0} \ldots b_{k} w_{j} \ldots w_{n}$ is the reduced sequence of $S$. Note that $H^{\prime}$ remains the same and $v(L) \subseteq v\left(L_{1}\right)$ (since $v_{i-1} \neq v_{i}$ ), but $J_{1}$ contains $J$ as a proper subgraph, contradicting (III). This proves (e).

Because (2) does not hold for $G$, we have

(f) For any $T \subseteq V\left(L^{*}\right)$ with $|T| \leq 3$, every component of $L^{*}-T$ contains a vertex in $V(A \cup C) \cup\left\{v_{0}, \ldots, v_{m}\right\}$.

By (a)-(f), $L^{*}, L, L^{\prime}, A, C$ satisfy all conditions of (2.5). Hence, by (2.5), $\left(L^{*},(a, b, c),\left(a^{\prime}, b^{\prime}, c^{\prime}\right)\right)$ is a ladder along a sequence $z_{0} z_{1} \ldots z_{p}$ whose reduced sequence is the reduced sequence of $v_{0} \ldots v_{m}$. Hence $w_{0} \ldots w_{n}$ is the reduced sequence of $z_{0} \ldots z_{p}$. Therefore, either $G=L^{*}$ or $G$ has a separation $\left(J, L^{*}\right)$ such that $V\left(J \cap L^{*}\right)=$ $\left\{w_{0}, \ldots, w_{n}\right\},\left(J, w_{0}, \ldots, w_{n}\right)$ is 3 -planar, and $\left(L^{*},(a, b, c),\left(a^{\prime}, b^{\prime}, c^{\prime}\right)\right)$ is a ladder along $z_{0} \ldots z_{p}$, where $z_{0}=b, z_{p}=b^{\prime}, w_{0} \ldots w_{n}$ is the reduced sequence of $z_{0} \ldots z_{p}$.

Proof of (1.3). Suppose $\left(G,\{a, c\},\left\{a^{\prime}, c^{\prime}\right\},\left(b, b^{\prime}\right)\right)$ is an obstruction, and assume that, for any $T \subseteq V(G)$ with $|T| \leq 3$, every component of $G-T$ contains an element of $\{a, b, c\} \cup\left\{a^{\prime}, b^{\prime}, c^{\prime}\right\}$. Then by (3.1), (1), (2) or (3) of (1.3) holds.

Now assume that (1), (2) or (3) of (1.3) holds. We wish to show that $\left(G,\{a, c\},\left\{a^{\prime}, c^{\prime}\right\},\left(b, b^{\prime}\right)\right)$ is an obstruction.

If (1) of (1.3) holds, then $\left(G,\{a, c\},\left\{a^{\prime}, c^{\prime}\right\},\left(b, b^{\prime}\right)\right)$ is an obstruction in the trivial sense. If (2) of (1.3) holds, then $\left(G,(a, b, c),\left(a^{\prime}, b^{\prime}, c^{\prime}\right)\right)$ is a ladder, and so, an obstruction. So we may assume that (1) and (2) of (1.3) do not hold, and (3) of (1.3) holds. Then $G$ contains three disjoint paths from $\{a, b, c\}$ to $\left\{a^{\prime}, b^{\prime}, c^{\prime}\right\}$. Let $A, B, C$ be disjoint paths in $G$ from $\{a, b, c\}$ to $\left\{a^{\prime}, b^{\prime}, c^{\prime}\right\}$, with $b \in B$. We may assume that $A, B, C$ are chosen so that $b^{\prime} \notin B$; for otherwise, $\left(G,(a, b, c),\left(a^{\prime}, b^{\prime}, c^{\prime}\right)\right)$ is an obstruction.

Let $(J, L)$ be a separation of $G$ such that $V(J \cap L)=\left\{w_{0}, \ldots, w_{n}\right\},\left(J, w_{0}, \ldots, w_{n}\right)$ is 3planar, and $\left(L,(a, b, c),\left(a^{\prime}, b^{\prime}, c^{\prime}\right)\right)$ is a ladder with rungs $\left(R_{i},\left(x_{i-1}, v_{i-1}, y_{i-1}\right),\left(x_{i}, v_{i}, y_{i}\right)\right)$, $i=1, \ldots, m$, where $x_{0}=a, v_{0}=b, y_{0}=c, x_{m}=a^{\prime}, v_{m}=b^{\prime}, y_{m}=c^{\prime}$, and $w_{0} \ldots w_{n}$ is the reduced sequence of $v_{0} \ldots v_{m}$. We use induction on the number of rungs in $L$.

Since $b^{\prime} \notin B, B \nsubseteq J$. Hence, let $w_{k} \in B$ with $k$ maximum such that $b B w_{k} \subseteq J$. Then $k<n$; otherwise, $b^{\prime} \in B$ (since $\left(J, w_{0}, \ldots, w_{n}\right)$ is 3-planar). Let $v_{s}=w_{k}$ with $s$ minimum and let $v_{t}=w_{k+1}$ with $t$ maximum. Let $L^{*}$ be the union of $\bigcup_{s<i \leq t} R_{i}$ and those edges of $L$ with both ends in some $\left\{x_{i}, v_{i}, y_{i}\right\}, s<i \leq t$. Then $\left(L^{*},\left(x_{s}, v_{s}, y_{s}\right),\left(x_{t}, v_{t}, y_{t}\right)\right)$ is 
a ladder along $v_{s} \ldots v_{t}$. Since every path in $G$ from $\{a, b, c\}$ to $\left\{a^{\prime}, b^{\prime}, c^{\prime}\right\}$ must intersect $V\left(b B w_{k}\right) \cup\left\{x_{s}, y_{s}\right\}, A \cap L^{*}, B \cap L^{*}$, and $C \cap L^{*}$ are disjoint paths from $\left\{x_{s}, w_{k}, y_{s}\right\}$ to $\left\{x_{t}, w_{k+1}, y_{t}\right\}$. Since $\left(L^{*},\left(x_{s}, v_{s}, y_{s}\right),\left(x_{t}, v_{t}, y_{t}\right)\right)$ is a ladder, $B \cap L^{*}$ is a path from $w_{k}$ to $w_{k+1}$.

Let $L^{\prime}$ denote the union of $\bigcup_{j=t+1}^{m} R_{j}$ and edges in $L$ with both ends in some $\left\{x_{j}, v_{j}, y_{j}\right\}, j=t+1, \ldots, m$. Then $\left(L^{\prime},\left(x_{t}, v_{t}, y_{t}\right),\left(a^{\prime}, b^{\prime}, c^{\prime}\right)\right)$ is a ladder along $v_{t} \ldots v_{m}$. Let $J^{\prime}=J+w_{k} w_{k+1}$, and let the edge $w_{k} w_{k+1}$ be added such that $\left(J^{\prime}, w_{0}, \ldots, w_{n}\right)$ is 3-planar. It is easy to see that $A \cap\left(J^{\prime} \cup L^{\prime}\right),\left(B \cap\left(J^{\prime} \cup L^{\prime}\right)\right)+w_{k} w_{k+1}, C \cap\left(J^{\prime} \cup L^{\prime}\right)$ are disjoint paths in $J^{\prime} \cup L^{\prime}$ from $\left\{b, x_{t}, y_{t}\right\}$ to $\left\{a^{\prime}, b^{\prime}, c^{\prime}\right\}$. Note that $\left(J^{\prime}, L^{\prime}\right)$ is a separation in $J^{\prime} \cup L^{\prime}$ such that $V\left(J^{\prime} \cap L^{\prime}\right)=\left\{b, w_{k+1}, \ldots, w_{n}\right\},\left(J^{\prime}, b, w_{k+1}, \ldots, w_{n}\right)$ is 3-planar, and $\left(L^{\prime},\left(x_{t}, b, y_{t}\right),\left(a^{\prime}, b^{\prime}, c^{\prime}\right)\right)$ is a ladder along $b v_{t} \ldots v_{m}$, where $v_{m}=w_{n}=b^{\prime}$, and $b w_{k+1} \ldots w_{n}$ is the reduced sequence of $b v_{t} \ldots v_{m}$. Since $L^{\prime}$ has fewer rungs than $L$, it follows from induction that $\left(B \cap\left(J^{\prime} \cup L^{\prime}\right)\right)+w_{k} w_{k+1}$ is from $b$ to $b^{\prime}$. Hence, $B$ is from $b$ to $b^{\prime}$. So $\left(G,\{a, c\},\left\{a^{\prime}, c^{\prime}\right\},\left(b, b^{\prime}\right)\right)$ is an obstruction.

\section{Connectivity}

In this section, we prove (1.4), and give an example to show that (1.4) is best possible.

Proof of (1.4). Suppose that $G$ is 8-connected and $\left\{a, b, c, a^{\prime}, b^{\prime}, c^{\prime}\right\} \subseteq V(G)$, and assume that $\left(G,\{a, c\},\left\{a^{\prime}, c^{\prime}\right\},\left(b, b^{\prime}\right)\right)$ is an obstruction. By (1.3), either (i) $\left(G,(a, b, c),\left(a^{\prime}, b^{\prime}, c^{\prime}\right)\right)$ is a ladder along a sequence $v_{0} \ldots v_{m}$ (in this case, let $J=$ $\left\{w_{0}, \ldots, w_{n}\right\}$ and $L=G$, where $w_{0} \ldots w_{n}$ is the reduced sequence of $v_{0} \ldots v_{m}$ ), or (ii) $G$ has a separation $(J, L)$ such that $V(J \cap L)=\left\{w_{0}, \ldots, w_{n}\right\},\left(J, w_{0}, \ldots, w_{n}\right)$ is 3-planar, and $\left(L,(a, b, c),\left(a^{\prime}, b^{\prime}, c^{\prime}\right)\right)$ is a ladder with rungs along $v_{0} \ldots v_{m}$, where $w_{0} \ldots w_{n}$ is the reduced sequence of $v_{0} \ldots v_{m}$.

Then $L-\left\{w_{0}, \ldots, w_{n}\right\} \neq \emptyset$; otherwise, $G=J$ is 3 -planar, and so, is at most 5connected, a contradiction. Since $\left(J, w_{0}, \ldots, w_{n}\right)$ is 3-planar and since $G$ is 8-connected, $J$ is a plane graph. Let $\left(R_{i},\left(x_{i-1}, v_{i-1}, y_{i-1}\right),\left(x_{i}, v_{i}, y_{i}\right)\right), i=1, \ldots, m$, be the rungs of $L$, where $x_{0}=a, v_{0}=b, y_{0}=c, x_{m}=a^{\prime}, v_{m}=b^{\prime}$, and $y_{m}=c^{\prime}$.

(a) We claim that $L$ is at most 6 -connected.

This is obvious when $m \geq 2$. So assume that $m=1$. Then $\left(L,(a, b, c),\left(a^{\prime}, b^{\prime}, c^{\prime}\right)\right)$ is a rung. Since $\{a, c\} \neq\left\{a^{\prime}, c^{\prime}\right\}$ and $b \neq b^{\prime}, L$ is at most 6 -connected.

By (a) and since $G$ is 8-connected, $J-\left\{w_{0}, \ldots, w_{n}\right\} \neq \emptyset, n \geq 7$, and $V\left(R_{i}\right)=$ $\left\{x_{i-1}, y_{i-1}, v_{i-1}\right\} \cup\left\{x_{i}, y_{i}, v_{i}\right\}$ for $i \in\{1, \ldots, m\}$.

(b) We claim that $v_{0} \neq v_{1}$ and $\left\{x_{1}, y_{1}\right\}=\left\{x_{0}, y_{0}\right\}$, and $v_{m-1} \neq v_{m}$ and $\left\{x_{m}, y_{m}\right\}=$ $\left\{x_{m-1}, y_{m-1}\right\}$.

If $\left\{x_{1}, y_{1}\right\} \neq\left\{x_{0}, y_{0}\right\}$, then $\left\{v_{0}, x_{1}, v_{1}, y_{1}\right\}$ is a cut set of $G$, a contradiction. So $\left\{x_{1}, y_{1}\right\}=\left\{x_{0}, y_{0}\right\}$, and hence, $v_{1} \neq v_{0}$. 
Similarly, $v_{m-1} \neq v_{m}$ and $\left\{x_{m}, y_{m}\right\}=\left\{x_{m-1}, y_{m-1}\right\}$.

(c) We claim that, for every $x \in V(J)-\left\{w_{1}, \ldots, w_{n-1}\right\}, x$ is adjacent to at most two vertices in $\left\{w_{1}, \ldots, w_{n-1}\right\}$.

Suppose that $x$ is adjacent to at least three vertices of $\left\{w_{1}, \ldots, w_{n-1}\right\}$. Let $i, j \in$ $\{1, \ldots, n-1\}$ with $i \leq j-2$ such that $w_{i}, w_{j}$ are neighbors of $x$. Let $v_{k}=w_{i}$ and $v_{l}=w_{j}$. Then $\left\{v_{k}, x_{k}, y_{k}, v_{l}, x_{l}, y_{l}, x\right\}$ is a cut set of $G$, a contradiction.

Now, consider $J^{\prime}=J-\left\{w_{1}, \ldots, w_{n-1}\right\}$. Since $J$ is a plane graph, $J^{\prime}$ is a plane graph. Let $x \in V\left(J^{\prime}\right)$. If $x \in\left\{w_{0}, w_{n}\right\}$, then by (b) and (c) (since $w_{0}=v_{0}$ and $w_{n}=v_{m}$ ), the degree of $x$ in $J^{\prime}$ is at least 3 . If $x \notin\left\{w_{0}, w_{n}\right\}$, then by (c), the degree of $x$ in $J^{\prime}$ is at least 6. Thus the number of edges in $J^{\prime}$ is at least $\frac{6\left(\left|V\left(J^{\prime}\right)\right|-2\right)+2 \cdot 3}{2}=3\left|V\left(J^{\prime}\right)\right|-3$, contradicting the planarity of $J^{\prime}$.

Next, we show that (1.4) is best possible by constructing 7-connected obstructions. A near triangulation is a plane graph in which every face, with the possible exception of the infinite face, is bounded by a triangle.

First, we construct a near triangulation $J$ whose outer cycle $C$ contains an edge $b_{1} b_{2}$ (with $E\left(b_{1} C b_{2}\right)=\left\{b_{1} b_{2}\right\}$ ) such that

(1) $d(x) \geq 3$ for all $x \in V(J)$,

(2) $d(x) \geq 7$ for all $x \in V(J-C) \cup\left\{b_{1}, b_{2}\right\}$,

(3) if $T$ is a cut set of $J$ with $|T| \leq 6$, then $|T| \geq 3$ and every component of $J-T$ contains a vertex of $C$, and

(4) for each $x \in V(C)-\left\{b_{1}, b_{2}\right\}$, there does not exist $T \subseteq V(J)$ with $|T| \leq 4$ such that every path from $b_{2} C x$ to $x C b_{1}$ in $J-b_{1} b_{2}$ intersects $T$.

We start with a path $J_{0}=P_{0}$ consisting of a single edge $b_{1} b_{2}$. Suppose we have constructed a near triangulation $J_{i}$ and a path $P_{i}$ in $J_{i}$ for some $i \geq 0$ such that $P_{0} \cap P_{i}=\emptyset$ and $P_{0} \cup P_{i}$ is contained in the outer cycle of $J_{i}$. We construct a near triangulation $L_{i+1}$ as follows. (See Figure 2 for an illustration with $i=3$.)

Let $P_{i+1}$ be a sufficiently long path (for example, at least 6 times as long as $P_{i}$ ). In the disjoint union of $P_{i+1}$ and $L_{i}$, we add edges from each vertex of $P_{i}$ to at least six consecutive vertices on $P_{i+1}$ so that $P_{i+1} \cup P_{0}$ is contained in the outer cycle of $J_{i+1}$, $d(x) \geq 7$ if $x \in V\left(J_{i}\right), d(x) \geq 3$ if $x \in V\left(P_{i+1}\right)$ and $x$ is not an end of $P_{i+1}$, and $d(x)=2$ if $x$ is an end of $P_{i+1}$.

Let $x$ and $x^{\prime}$ be the ends of $P_{4}$. Add a sufficiently long path $Q$ from $x$ to $x^{\prime}$ such that $Q$ is internally disjoint from $J_{4}$. We add edges from each vertex of $P_{4}-\left\{x, x^{\prime}\right\}$ to at least six consecutive vertices on $Q$ such that the result is a near triangulation $J, Q \cup P_{0}$ is contained in its outer cycle, and $d(x) \geq 7$ if $x \in V\left(J_{4}\right)$ and $d(x) \geq 3$ if $x \in V(Q)$. It is 
straightforward to check that the resulting graph $J$ satisfying (1)-(4) above. We relabel the vertices in $J$ so that $C=w_{0} w_{1} \ldots w_{n} w_{0}$ is the outer cycle of $J$, where $w_{0}=b_{2}=b$ and $w_{n}=b_{1}=b^{\prime}$. See Figure 2. We may choose $n$ to be odd.

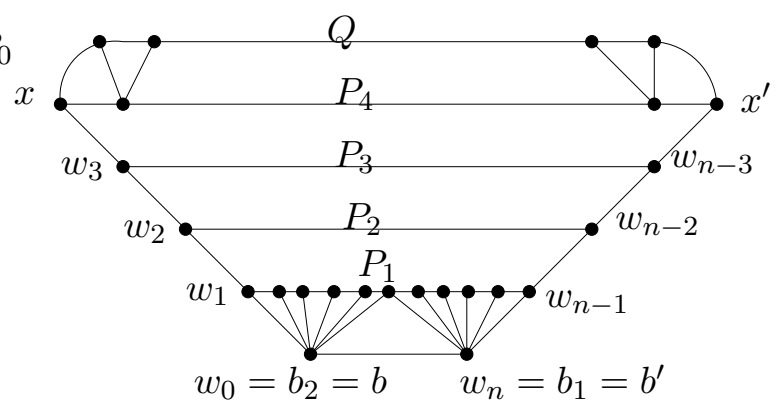

Figure 2: A near triangulation.

Next, we construct a ladder along $v_{0} \ldots v_{m}$ whose reduced sequence is $w_{0} \ldots w_{n}$, where $m=2 n-3$ and $n \geq 6$ is an odd integer, and describe how it is attached to $J$ along $w_{0} \ldots w_{n}$. See Figure 3 .

Let $v_{i}=w_{i}$ for $i=0,1,2$, and let $v_{m-2}=w_{n-2}, v_{m-1}=w_{n-1}$, and $v_{m}=w_{n}$. For $i=1,2, m-1, m$, let $x_{i-1}=x_{i}$ and $y_{i-1}=y_{i}$, and let $R_{i}^{\prime}$ be the complete graph on $\left\{x_{i}, y_{i}, v_{i-1}, v_{i}\right\}$. For each $i=2 k+1,3 \leq i \leq m-2$, let $x_{i-1} \neq x_{i}, y_{i-1} \neq y_{i}$, and $v_{i-1}=v_{i}=w_{k+1}$, and let $R_{i}^{\prime}$ be the complete graph on $\left\{x_{i-1}, x_{i}, v_{i}, y_{i-1}, y_{i}\right\}$. For each $i=2 k+2$ with $4 \leq i \leq m-3$, let $x_{i-1}=x_{i}, y_{i-1}=y_{i}, v_{i-1}=w_{k+1}$ and $v_{i}=w_{k+2}$, and let $R_{i}^{\prime}$ be the complete graph on $\left\{x_{i}, v_{i-1}, v_{i}, y_{i}\right\}$. Let $S$ be the set of 2-element subsets of $\left\{x_{i}, v_{i}, y_{i}\right\}$ for all $i=0, \ldots, m$. Let $R_{i}=R_{i}^{\prime}-S$. Then $\left(R_{i},\left(x_{i-1}, v_{i-1}, y_{i-1}\right),\left(x_{i}, v_{i}, y_{i}\right)\right)$ is a rung. Let $L=\left(\bigcup_{i=1}^{m} R_{i}\right)+S$. Then $\left(L,\left(x_{0}, v_{0}, y_{0}\right),\left(x_{m}, v_{m}, y_{m}\right)\right)$ is a ladder.

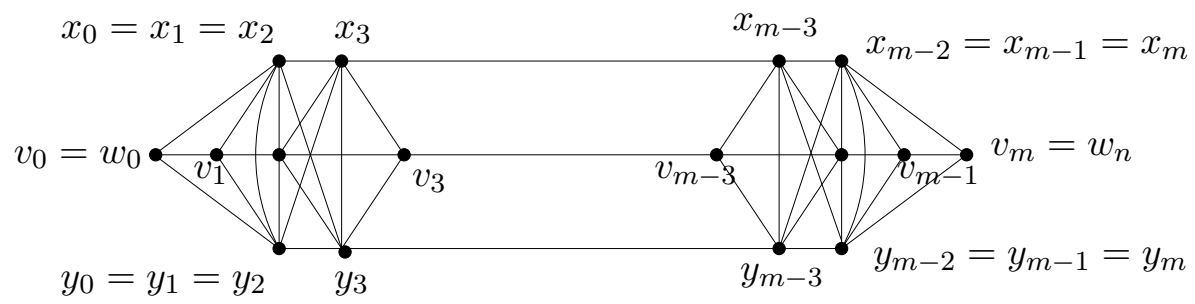

Figure 3: The ladder $L$.

Finally, let $G$ be obtained from the disjoint union of $J$ and $L$ by identifying the vertices $w_{0}, \ldots, w_{n}$ in both $J$ and $L$. Now $(J, L)$ is a separation in $G, V(J \cap L)=\left\{w_{0}, \ldots, w_{n}\right\}$, $\left(J, w_{0}, \ldots, w_{n}\right)$ is 3 -planar, and $\left(L,\left(x_{0}, v_{0}, y_{0}\right),\left(x_{m}, v_{m}, y_{m}\right)\right)$ is a ladder along $v_{0} v_{1} \ldots v_{m}$, 
where $w_{0} \ldots w_{n}$ is the reduced sequence of $v_{0} \ldots v_{m}$.

By (1.3), $\left(G,\left\{x_{0}, y_{0}\right\},\left\{x_{m}, y_{m}\right\},\left(v_{0}, v_{m}\right)\right)$ is an obstruction.

We claim that $G$ is 7 -connected. Suppose on the contrary that $G$ is not 7-connected. Then $G$ contains a cut set $T$ with $|T| \leq 6$. Let $X$ be a component of $G-T$. If $V(X) \cap\left\{w_{0}, \ldots, w_{n}\right\}=\emptyset$, then $X \subseteq L-\left\{w_{0}, \ldots, w_{n}\right\}$ (since $|T| \leq 6$ and by (3)), and so, $|T| \geq 7$ (by the construction of $L$ ), a contradiction. So $V(X) \cap\left\{w_{0}, \ldots, w_{n}\right\} \neq \emptyset$.

If $(V(X) \cap V(L))-\left\{w_{0}, \ldots, w_{n}\right\} \neq \emptyset$, then $|T \cap V(L)| \geq 7$ (by the construction of $L)$, a contradiction.

Hence, $V(X) \cap V(L) \subseteq\left\{w_{0}, \ldots, w_{n}\right\}$, and so, $X$ is a component of $J-(T \cap V(J))$ and the neighbors of $V(X) \cap\left\{w_{0}, \ldots, w_{n}\right\}$ in $L$ must be contained in $T$. By (3), $\mid T \cap$ $V(J) \mid \geq 3$. If $V(X) \cap\left\{w_{2}, \ldots, w_{n-2}\right\} \neq \emptyset$, then $\left|(T \cap L)-\left\{w_{0}, \ldots, w_{n}\right\}\right| \geq 4$, and so, $|T \cap V(J)| \leq 2$, a contradiction. So assume that $V(X) \cap V(L) \subseteq\left\{w_{0}, w_{1}, w_{n-1}, w_{n}\right\}$. Furthermore, $V(X) \cap V(L) \subseteq\left\{w_{0}, w_{1}\right\}$ or $V(X) \cap V(L) \subseteq\left\{w_{n-1}, w_{n}\right\}$; as otherwise, $\left|(T \cap V(L))-\left\{w_{0}, \ldots, w_{n}\right\}\right| \geq 4$, and so, $|T \cap V(J)| \leq 2$, a contradiction. So there is some $x \in V\left(b_{2} C b_{1}\right)$ such that every path from $b_{2} C x$ to $x C b_{1}$ in $J-b_{1} b_{2}$ intersects $T \cap V(J)$. Since $\left|(T \cap V(L))-\left\{w_{0}, \ldots, w_{n}\right\}\right| \geq 2$, and so, $|T \cap V(J)| \leq 4$, contradicting (4).

Seymour pointed out that (1.3) can be used to solve the following problem: Let $G$ be a graph and $\left\{b, b^{\prime}, x, y\right\} \subseteq V(G)$; determine if $G$ contains a path from $b$ to $b^{\prime}$ and through $x$ and $y$ in that order.

To see this, we consider two cases. If $x y \in E(G)$, then this problem reduces to the problem for finding a $\left(\{b, x\},\left\{b^{\prime}, y\right\}\right)$-linkage in $G$, and so, can be solved using (2.1). So assume that $x y \notin E(G)$. Let $G^{\prime}$ be the graph obtained from $G$ by replacing $x$ with vertices $a^{\prime}, c^{\prime}$ and joining $a^{\prime}, c^{\prime}$ to all neighbors of $x$, and replacing $y$ with vertices $a, c$ and joining $a, c$ to all neighbors of $y$. It is easy to verify that $G$ contains a path from $b$ to $b^{\prime}$ through $x$ and $y$ in that order iff $G^{\prime}$ contains three disjoint paths from $\{a, b, c\}$ to $\left\{a^{\prime}, b^{\prime}, c^{\prime}\right\}$ with no path from $b$ to $b^{\prime}$.

Another problem mentioned to me by Seymour is to characterize the connected graphs containing cycles through four given vertices in a prescribed order. Using a similar technique as above, this problem can be formulated as follows. Let $G$ be a graph, $\left\{a_{1}, a_{2}, b_{1}, b_{2}\right\} \subseteq V(G)$, and $\left\{c_{1}, c_{2}, d_{1}, d_{2}\right\} \subseteq V(G)$. Characterize those $G$ such that, for any four disjoint paths from $\left\{a_{1}, a_{2}, b_{1}, b_{2}\right\}$ to $\left\{c_{1}, c_{2}, d_{1}, d_{2}\right\}$, the two paths with an end in $\left\{a_{1}, a_{2}\right\}$ have their other ends both in $\left\{c_{1}, c_{2}\right\}$ or both in $\left\{d_{1}, d_{2}\right\}$. The planar case is solved in ([4], Theorem 2.1).

\section{ACKNOWLEDGMENT}

I would like to thank Robin Thomas for bringing this problem to my attention. I also would like to thank Paul Seymour for pointing out the connection between the main result of this paper and the problems about paths and cycles through vertices in a specific order. 


\section{References}

[1] K. Chakravarti and N. Robertson, Covering three edges with a bond in a nonseparable graph. Annals of Discrete Math. (Deza and Rosenberg eds) (1979) 247.

[2] P. D. Seymour, Disjoint paths in graphs. Discrete Math. 29 (1980) 293-309.

[3] C. Thomassen, 2-Linked graphs. Europ. J. Combin. 1 (1980) 371-378.

[4] X. Yu, Subdivisions in planar graphs. J. Combin. Theory, Ser. B 72 (1998) 10-52.

[5] X. Yu, Disjoint paths in graphs I, 3-planar graphs and basic obstructions. Annals of Combinatorics 7 (2003) ***_***.

[6] X. Yu, Disjoint paths in graphs II, a special case. Annals of Combinatorics 7 (2003) $* * * \_* * *$. 